\title{
Keltischer oder etruskischer Einfluss? \\ Ein späthallstattzeitlicher Armreif \\ mit gegenständiger Kopfzier aus Möllbrücke (Kärnten)
}

\author{
Paul Gleirscher
}

\begin{abstract}
Zusammenfassung
Auf einem namenlosen Höhenrücken bei Möllbrücke westlich von Spittal/Drau wurde 2012 ein bronzener Armreif mit doppelter gegenständiger Kopfzier gefunden. Es könnte sich um eine kultische Deponierung handeln. Der Armreif gehört zur Gruppe der massiven Knotenarmreifen der Späthallstattzeit und datiert ins ausgehende 6. oder 5. Jh. v. Chr. Für Arm- und Fußringe mit Kopfzier lassen sich eine Reihe an Vergleichsfunden beibringen, insbesondere aus dem Bereich des frühkeltischen Kulturkreises. Kopf- bzw. Gesichtsappliken („Masken“) vergleichbarer Zeitstellung sind aber auch im südalpinen Raum in unterschiedlichen Ausformungen belegt. Während sie in Oberitalien als etruskischer Einfluss gelten, sieht die slowenische Forschung darin - insbesondere mit Blick auf zwei Fingerringe aus Vače - einen frühen keltischen Einfluss. Weil auch der Südostalpenraum damals wesentliche Impulse seitens der etruskischen Kultur erfahren hat, ist aber auch ein direkter mediterraner Einfluss für das Aufkommen der Kopfzier zu erwägen. Der Armring aus Möllbrücke ist vom Typ her jedenfalls ein lokales Produkt. Gesichtsappliken gelten zum einen als Ausdruck apotropäischer Vorstellungen, zum anderen als Bilder von Göttern, Heroen und Dämonen. Außerdem werden in ihnen symbolisch Köpfe von im Kampf getöteten Kriegern gesehen (têtes coupées).
\end{abstract}

\section{Schlüsselbegriffe}

Armreif, Späthallstattzeit, Südostalpenraum, Kopf-/Gesichtsappliken („Masken“), keltisch, etruskisch.

Abstract - Celtic or Etruscan Influence? A Late Hallstatt Period Bracelet with Opposing Head Applications from Möllbrücke (Carinthia)

On a nameless ridge near Möllbrücke west of Spittal/Drau a bronze bracelet with double opposing head applications was found in 2012 . It is likely to be a cultic deposit. The bracelet belongs to the group of the massive bracelets with knots (Knotenarmreifen) of the Late Hallstatt period and dates to the later $6^{\text {th }}$ or $5^{\text {th }}$ century BC. There are numerous comparable objects for bracelets and foot rings with a head application, especially in early Celtic art, but contemporaneous applications in the form of heads or faces ('masks') are also known in the southern Alpine area in different forms. While they are regarded as an Etruscan influence in northern Italy, Slovenian researchers detect an early Celtic influence, especially with regard to two finger rings from Vače. However, it seems that a direct Mediterranean influence for the emergence of the decoration with heads/faces ('masks') has to be considered as well, as significant influence from the Etruscan civilization reached the southeastern Alpine area at that time. The bracelet from Möllbrücke is a local product. Applications in the form of heads/faces ('masks') are regarded as an expression of apotropaic ideas on the one hand, and as images of gods, heroes and demons on the other. Additionally, they are interpreted as symbols for the heads of warriors who were killed in combat (têtes coupées).

\section{Keywords}

Bracelet, Late Hallstatt period, southeastern Alpine area, applications in the form of heads/faces ('masks'), Celtic, Etruscan.

\section{Fundort und Beschreibung}

Auf ihrem Weg von Lienz nach Spittal trennt die Drau die Gailtaler Alpen im Süden von der Kreuzeckgruppe im Norden. Kurz vor Spittal erreicht sie bei Sachsenburg eine Talenge, ehe sie ins Lurnfeld gelangt, wo die Möll mündet. Ausgehend vom Salzkogel (2493 m ü. NN) erreicht hier ein langgezogener Höhenrücken das Lurnfeld. Vis-à-vis befindet sich am Südufer der Drau die in napoleonischer Zeit zerstörte mittelalterliche Festung der Salzburger Erzbischöfe, die ältere „Vorläufer“ haben dürfte. ${ }^{1}$ Teurnia in keltischer und römischer Zeit (heute St. Peter in Holz) ${ }^{2}$ und später das unweit östlich gelegene Spittal verdanken ihre zentralörtliche Bedeutung ihrer Lage an der Drau, an den Routen nach Norden über die Tauernpässe, vom Hochtor über den Mallnitzer Tauern bis zum Katschberg.

\footnotetext{
1 Kohla 1973, 282-283.

2 Zusammenfassend, mit Blick auch auf die Fundstätten im Umland: Glaser 1992. - Glaser 2002. - Vgl. zur spätantiken Stadt und zum Übergang ins Frühmittelalter auch Gleirscher 2018b, 22-27, 34-37, 86-89, 114-115, 122-126, 230-231, 243-245, 285-286.
} 
Auf dem genannten namenlosen Höhenrücken zwischen Sachsenburg und Möllbrücke (Abb. 1) hat Kurt Scheuch sen. aus Mühldorf, dem für das Überlassen des Objektes zur wissenschaftlichen Untersuchung herzlich gedankt sei, einen außergewöhnlichen Armreifen aus massiver Bronze gefunden. Die Fundstelle liegt nordseitig am Grat, rund $250 \mathrm{~m}$ über dem Talboden. Im Gelände zeigen sich heute keinerlei Auffälligkeiten. Der Höhenrücken ist relativ schmal und bietet beste Ausblicke ins Drautal nach Sachsenburg, ins untere Mölltal und ins Lurnfeld. Wiederholt zeigen sich Felsausbisse mit Gletscherschliff, mitunter schroffe Abbruchkanten. Ansatzweise lassen sich an verschiedenen Stellen „Verebnungen“ ausmachen, an einer bereits höher gelegenen Stelle „Terrassierungen“ und eine Art Wallkante. ${ }^{3}$ An der Fundstelle des Armreifens (Quote ca. $800 \mathrm{~m}$ ü. NN), die auf Höhe des Draukraftwerkes von Sachsenburg liegt, haben sich weder beim Finden noch im Rahmen einer Begehung Hinweise auf weitere zugehörige Funde oder auf einen Befund, etwa Spuren eines verschliffenen Brandgrabes oder auf einen Kultplatz mit regelmäßig geübten, noch heute nachweisbaren Deponierungen, ergeben.

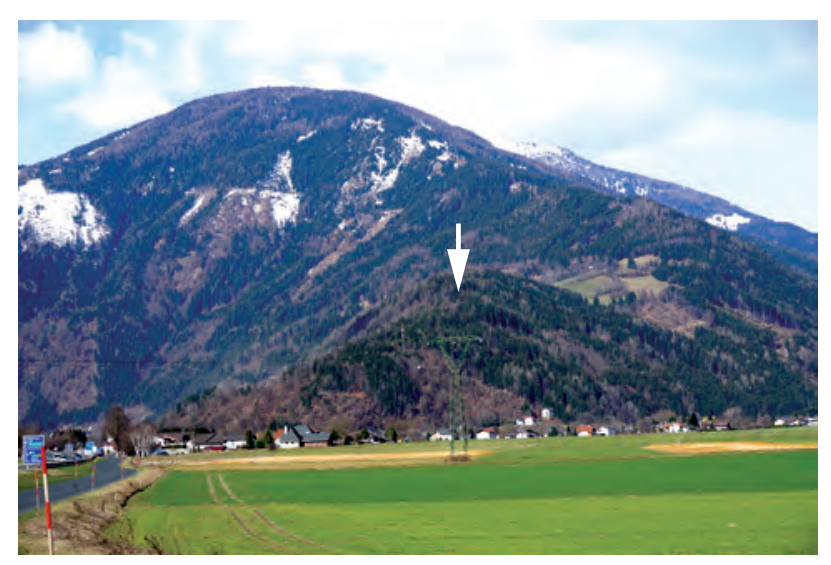

Abb. 1. Höhenrücken zwischen dem Drautal und dem Mölltal westlich von Spittal an der Drau (Foto: P. Gleirscher).

Der Armreif stammt, soweit die Nachuntersuchung Aussagen zulässt, offenbar aus keinem Grab und jedenfalls nicht aus einer Siedlung. Einer Deutung als Verlustfund steht somit eine Einschätzung als Einzeldeponierung mit religiösem Hintergrund gegenüber. ${ }^{4}$ Träfe Letzteres zu, ließe er sich der Gruppe jenes Ringschmuckes anschließen, der

3 Vermutlich angesprochen bei KoHLa 1973, 218.

4 U. a. STÖLlnER 2002. im Verlauf der jüngeren Eisenzeit aus kultischen Gründen vergraben wurde. In ihrer schillerndsten Variante wird diese Form der Deponierung im Alpenraum am Hortfund aus Erstfeld (Kanton Uri) sichtbar, wo man 1962 im Lochertal an der Route über den St. Gotthardpass im Zuge von Arbeiten zur Wildbachverbauung gleich auf sieben neuwertige Arm- und Halsreifen aus Gold gestoßen ist, die unter einem Felsblock niedergelegt worden waren. ${ }^{5}$ Sie weisen zugleich Merkmale von Goldbarren auf, was sich aus der pro Stück verwendeten und normierten Goldmenge erklärt. Es dürfte sich um die Bestückung eines oder mehrerer hölzerner keltischer Kultbilder aus der Zeit um 400 v. Chr. (ausgehende Stufe La Tène A) handeln. Einer der drei Armreifen zeigt eine typisch keltische Maskenzier.

Der Armreif aus Möllbrücke (Abb. 2) hat einen äußeren Durchmesser von 7,3 cm. Er ist durchschnittlich 0,7 cm breit und 0,5 cm stark, die lichte Weite beträgt $6,3 \mathrm{~cm}$. Er besteht aus massiver Bronze, wurde im Guss in verlorener Form hergestellt und ist dementsprechend nachgearbeitet. Der Querschnitt ist D-förmig. Die Verzierung des Armreifens ist völlig symmetrisch aufgebaut. Gegenüberliegend bestimmen zwei, durch einen Knoten und zwei an diesen anschließende, feine Rippen getrennte und Kopf-an-Kopf angebrachte Köpfe das Erscheinungsbild des Armreifens. Je zwölf Knoten zieren den Armreif zwischen den beiden Kopfgruppen, wobei zwischen den Knoten jeweils zwei feine Rippen herausgearbeitet sind. Die vier, in zwei Gruppen jeweils gegenständig angeordneten Köpfe sind überaus gleichförmig gearbeitet. Drei davon sind $1 \mathrm{~cm}$ lang, einer $1,2 \mathrm{~cm}$. Haare bzw. Frisur sind durch einen Wulst mit zum Scheitel hin gegenständig geführten Querkerben wiedergegeben, die Augen durch schräg nach unten führende, über der Nase zusammenstoßende Schlitze. Deutlich plastisch herausgearbeitet ist die Nase. Der leicht geöffnete, waagrechte Mund schließt das Gesicht jeweils zum markant gearbeiteten Kinn hin ab. Die einfache und gleichartige Ausführung aller vier Köpfe besticht durch ihre Reduktion ebenso wie durch den maskenhaften Gesichtsausdruck.

Der Armreif aus Möllbrücke gehört zur Gruppe der massiven Knotenarmreifen oder geperlten Armreifen mit scheibenförmiger Zwischenzier und datiert demnach in die ausgehende Hallstattkultur. Diese umfasst im Südostalpenraum nach der auf Stane Gabrovec zurückgehenden Definition $^{6}$ die Stufe der Certosafibeln (entspricht Hallstatt D2/3) und die Stufe der Negauer Helme (entspricht La Tène A$\mathrm{B} 1)$; beide werden in einen älteren und in einen jüngeren Abschnitt unterteilt. Dendrochronologische Messungen

5 Ausführlich Guggisberg 2000.

6 Zuletzt Gabrovec 1987. 


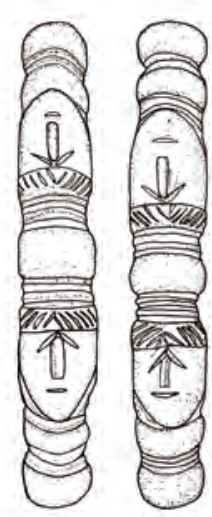

a $\quad b$

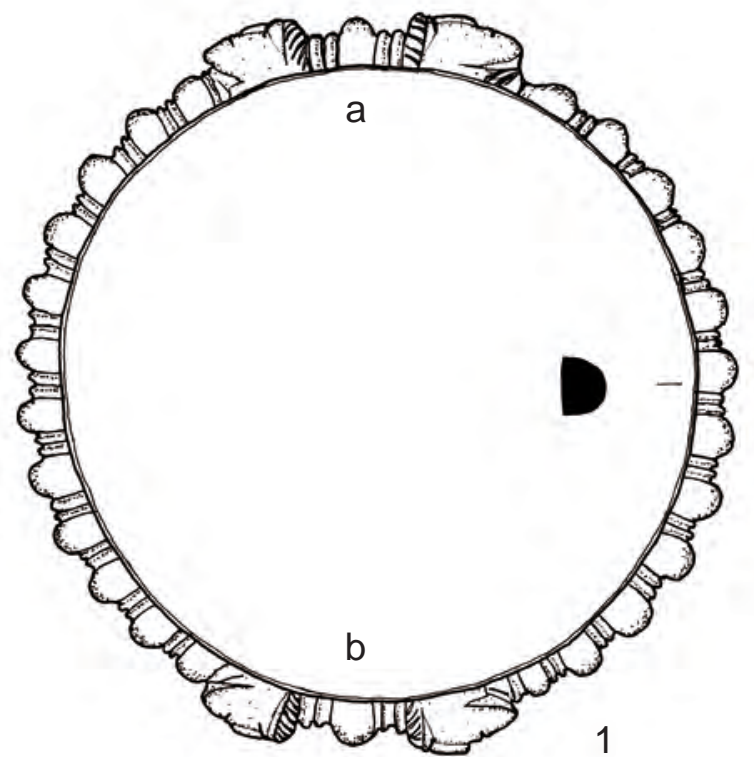

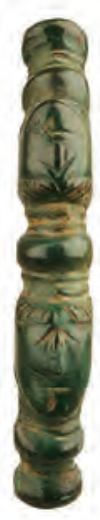
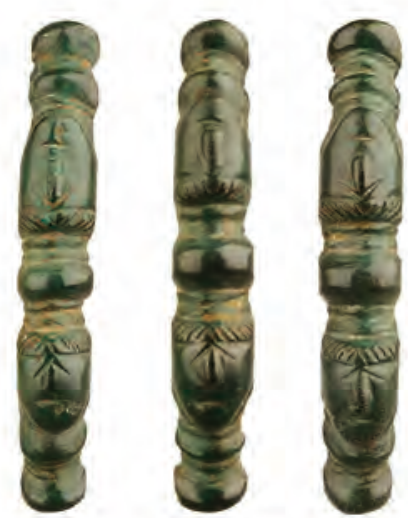

a

b

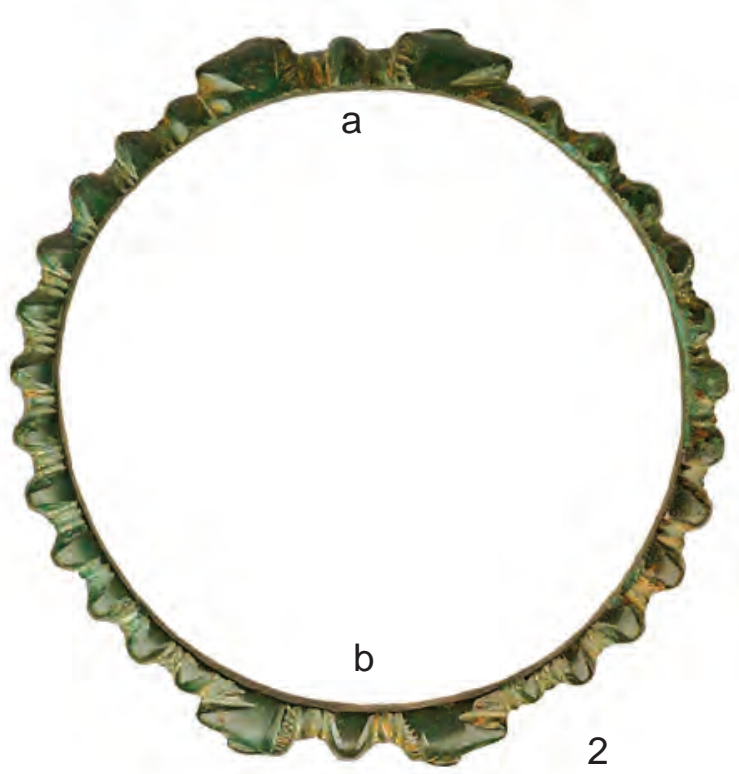

Abb. 2. Armreif aus Möllbrücke. Bronze, Maßstab 1:1 (Zeichnung: H. Mühlbacher, Foto: K. Allesch, beide Landesmuseum für Kärnten).

an den Bauhölzern der Grabkammer in Grabhügel $352 \mathrm{im}$ Areal Hallersbichl am Dürrnberg bei Hallein haben ergeben, dass diese im Herbst des Jahres 464 v. Chr. geschlagen wurden. 7 Weil das aussagekräftige Inventar des Grabes noch der Stufe Hallstatt D3 zuzurechnen ist und am Dürrnberg Latène A-zeitliche Funde bestens vertreten sind, kann der Beginn der Stufe Latène A nicht vor 460/450 v. Chr. angesetzt werden. Damit hat sich auch jene Diskrepanz aufgelöst, die bezüglich des ersten Auftretens von durchbrochenen Gürtelhaken oder Fühlatènefibeln zwischen Oberitalien und dem keltischen Kerngebiet nördlich der Alpen eine zeitliche Verschiebung von bis zu fünfzig Jahren angesetzt hatte.

7 Sormaz, Stöllner 2005, 364-365 und Abb. 4; 367-372. - Zum Grab: EGG, ZeLLer 2005.
Aus Slowenien liegt für den Südostalpenraum zur absolutchronologischen Einordnung der Certosa-Stufe (entspricht Stufe Hallstatt D2/3) ${ }^{8}$ mittlerweile die ${ }^{14} \mathrm{C}$-Datierung eines Oberschenkelknochens aus Grab 2 im Grabhügel von Grofove njive bei Drnovo südlich von Krško vor. Sie ergab ein kalibriertes Alter zwischen ca. 540 und 410 v. Chr. ${ }^{9}$ Und eine ${ }^{14} \mathrm{C}$-Datierung weist ein überaus reich ausgestattetes Kriegergrab aus Novo mesto/Kandija (Hügel IV/Grab 3) bzw. mit diesem den Horizont der Negauer Helme (entspricht La Tène A-B1) ${ }^{10}$ nunmehr in die Zeit zwischen 375 und 350 v. Chr. ${ }^{11}$ Das Ende der Späthallstattkultur bzw.

\footnotetext{
8 Teržan 1976, 437. - Dular 2003, 136-143.

9 Pavlovič 2014. - TeržAn, Črešnar 2014, 720-721.

10 TeržAn 1976, 439-442. - Dular 2003, 143-150.

11 Dular 2003, 144 und Abb. 87/5-6.
} 
der Beginn der Keltenzeit wird in Unterkrain (Dolenjsko) demnach zwischen ca. 350 und 325 v. Chr. angesetzt. ${ }^{12}$ Der massiv gefertigte, geschlossene Armreif mit Knotenzier und scheibenförmiger Zwischenzier aus Möllbrücke stellt hinsichtlich der Grundform ein lokales Produkt dar. ${ }^{13}$ Arm- und Fußreifen mit Knotenzier zählen in offener und geschlossener Ausführung bzw. bei vergleichbarer Formgebung auch in blecherner Ausführung zu den charakteristischen Schmuckstücken der Späthallstattkultur im Südostalpenraum.

In einem Frauengrab der Zeit um 550/500 v. Chr. aus Lopanec bei Kaplja vas (Hügel 2/Grab 1$)^{14}$ fanden sich zwei massive perlverzierte Fußreifen (lichte Weite $10 \mathrm{~cm}$ ) zusammen mit einer älteren Fibel und einem ebenso älteren Paar offener gerippter Armreifen mit sich überlappenden, spitz zulaufenden Enden. In der Certosa-Stufe treten dem Armreifen aus Möllbrücke im weiteren vergleichbare Armreifen auf, beispielsweise im Hügelgräberfeld Znančeve njive in Novo mesto. Anstelle der schmalen scheibenförmigen Zwischenzier haben die Armreifen aus Grab 8 in Hügel 2 allerdings eine perlverzierte Zwischenscheibe, ${ }^{15}$ während die Fußreifen aus demselben Frauengrab allein eine glatte Knotenzier aufweisen. ${ }^{16}$ Die Certosafibel erhellt eine frühe Stellung des Grabes innerhalb der Certosa-Stufe, demnach während der 2. Hälfte des 6. Jhs. v. Chr. Unweit von Vače wurden in Velika Kostrevnica mehrere Körpergräber beobachtet. Darf man den überlieferten Grabinventaren Glauben schenken, so fanden sich in einem in den Certosabzw. frühen Negauer-Horizont (2. Hälfte 6./5. Jh. v. Chr.) zu datierenden Grab Reiterelemente und auch ein massiver geperlter Fuß- oder Armreif mit zarten Zwischenrippen, ${ }^{17}$ trotz Scharnierkonstruktion jenem aus Möllbrücke vergleichbar.

Im weiteren vergleichbare, allerdings nicht geschlossene Arm- (lichte Weite ca. $6 \mathrm{~cm}$ ) und Fußringe (lichte Weite ca. $10 \mathrm{~cm}$ ) stammen mit hoher Wahrscheinlichkeit aus gestörten späthallstattzeitlichen Gräbern am Sattel unterhalb des Ajdovski gradec bei Vranje. ${ }^{18}$ Das Muster aus halbkugeligen Knoten („Perlen“) wird von feinen doppelten Rippen unterbrochen. Das kleine Fragment eines Armreifens aus Grab 18 von Paularo in Friaul zeigt Knoten und eine scheibenförmige Zwischenzier; das Frauengrab datiert um die

12 TERŽAN im Druck.

13 Für Hinweise danke ich D. Božič, Ljubljana.

14 Dular 2003, 262 und Taf. 84/5-6.

15 Typ IVc nach Tecco Hvala 2012, 301 und Abb. 111/12.

16 Dular 2003, 136 und Abb. 80A/6-7 (Armreifen), 16-17 (Fußreifen).

17 TERŽAN 2009, 88 und Abb. 5/B5.

18 Logar 1980, 298 und Abb. 2/2-5; 3/3-5.
Mitte des 6. Jhs. v. Chr. ${ }^{19}$ Dem sind zwei weitere Fragmente aus dem Gräberfeld in San Pietro al Natisone anzuschlieBen. ${ }^{20}$

\section{Vergleiche zur Zier}

Verschiedene Typen zeitgleicher Arm- und Fußreifen sowie Fingerringe zieren mitunter Kopfappliken. Ein großer, dünner, massiver Arm- oder Fußreifen aus Bronze (Abb. 3/3) stammt den Angaben des Finders zufolge aus einem Männergrab in Volčje nijve bei Mokronog. ${ }^{21}$ Biba Teržan tendiert zur Einschätzung, wonach es sich um einen geschlossenen Grabfund aus der Späthallstattzeit (Stufe Certosa bzw. Stufe der Negauer-Helme) handelt.22 In starker Stilisierung zeigt der Ring vier gegenständig angeordnete Doppelköpfe, von denen Augen und Ansätze einer Frisur zu erkennen sein dürften. Die starke Stilisierung erinnert an die Köpfe bzw. Gesichter auf einem Fußring aus dem La Tène A-zeitlichen Grab 2 in Hügel VII von Beuren/Kupp (Abb. 4/2) ${ }^{23}$ sowie auf einem Halsring aus dem La Tène Azeitlichen Grab 2 in Hügel IX von Theley (Abb. 4/3) ${ }^{24}$, beide im Saarland gelegen.

Ein bronzener Armreif mit zwei stark stilisierten, gegenständigen Kopfdarstellungen wurde auch im La Tène A-zeitlichen Grab 7 von Schwierberdingen (Abb. 3/1) in Baden-Württemberg, im Übrigen zusammen mit einer bemerkenswerten Maskenfibel (vgl. Abb. 9/7) gefunden;25 Carola Metzner-Nebelsick vermutet für die Trägerin rituelle Kompetenzen. ${ }^{26}$ Zwei gewissermaßen gegenständig angeordnete, dem Armreif aus Schwieberdingen an die Seite zu stellende Köpfe zieren einen spiralförmigen bronzenen Armreif, der aus Grab 2 in Grabhügel III von Novo mesto-Kandija (Abb. 3/4) stammt und dort unter anderem mit einem vergleichbaren, in stilisierten Schlangenköpfen endenden Armreif vergesellschaftet war. ${ }^{27}$ Wie am Stück aus Möllbrücke findet sich die gegenständige Kopfdarstellung auf einem südalpinen Typ von Armreifen, der wohl als venetisch anzusprechen ist. Die Gesichter der kleinen Köpfe auf dem Armreifen aus Novo mesto sind durch einfache

19 Vitri 2001, 28-29 und Abb. 8/12. - Corazza, Vitri 2001, 49 und Abb. 52.

20 Pettarin 2006, Taf. 21/327-328.

21 TeržAn 2009, 88 und Abb. 6/4. - B. Teržan, Ljubljana, hat das Stück dankenswerter Weise zwischenzeitlich überprüft und auch einen vierten Doppelkopf identifiziert.

22 TeržAn 2009, 88.

23 Cordie-Hackenberg 1992a, 146 und Abb. 1/2.

24 Cordie-Hackenberg 1992b, 174-175 und Abb. 5.

25 Stroh 1935, 292-293. - Binding 1993, 54-55 und Abb. 17/2.

26 Metzner-Nebelsick 2007, 717 und Abb. 4/5-6.

27 Knez 1986, 83-84 und Taf. 23/21. - TerŽAn im Druck, Abb. 13-

14. 


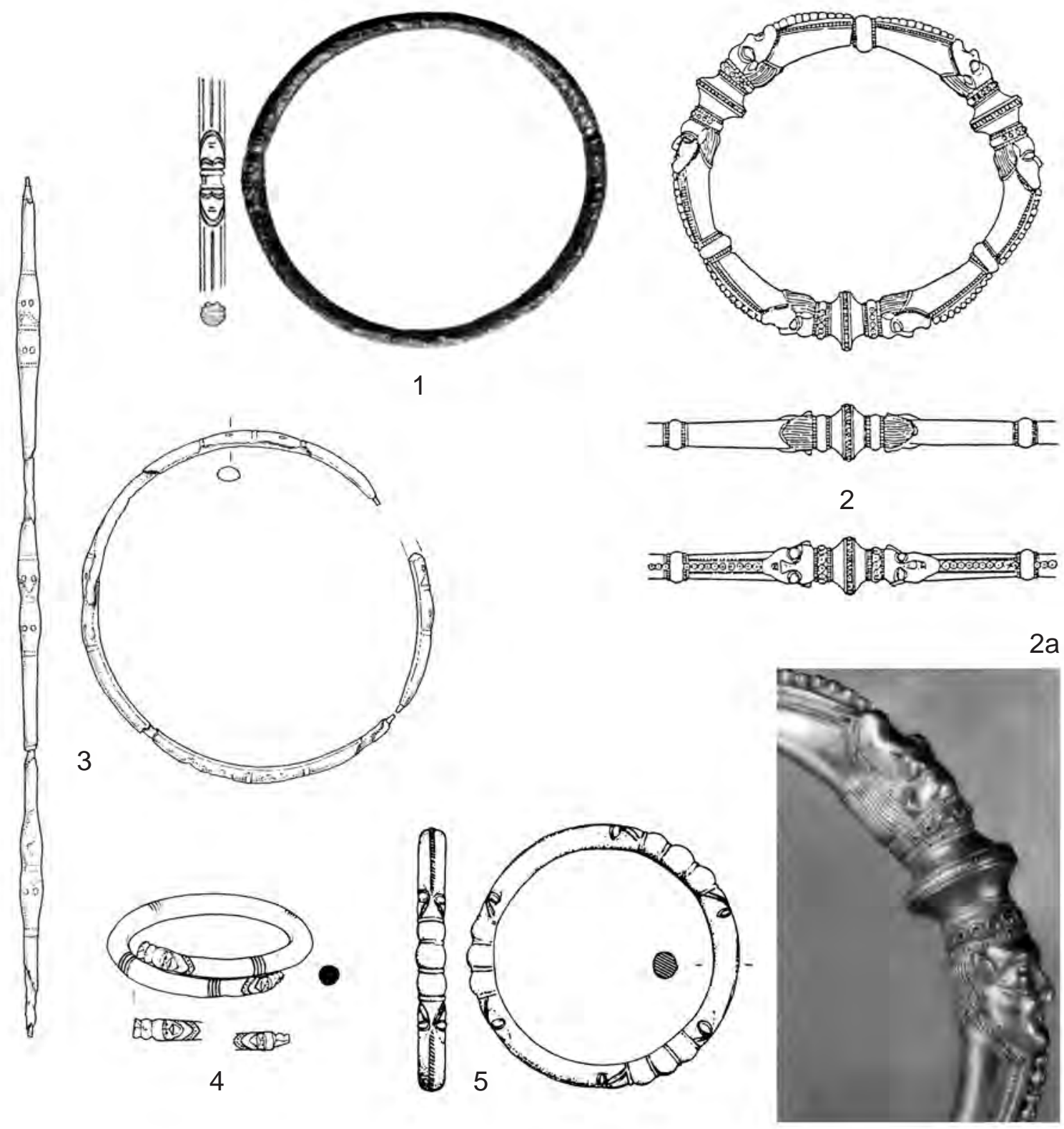

Abb. 3. Kopfdarstellungen auf frühlatènezeitlichem Ringschmuck. - 1. Schwieberdingen. Bronze, Maßstab 1:2 (METZNERNebelsick 2007, 717 und Abb. 4/5-6). - 2. Bad Dürkheim. Gold, Maßstab 1:2 (JoAchim 1992, 182 und Abb. 1). - 2a. Bad Dürkheim. Gold, Maßstab 2:1 (Megaw, Megaw 1989, 73 und Abb. 78). - 3. Volčje njive. Bronze, Maßstab 1:2 (TeržAn 2009,88 und Abb. 6/4). - 4. Novo mesto-Kandija, Hügel III/Grab 2. Bronze, Maßstab 1:2 (Knez 1986, 83-84 und Taf. 23/21). - 5. Speikern. Bronze, Maßstab 1:2 (Binding 1993, 90 und Abb. 34/9).

geometrische Gravur kenntlich gemacht. Ob sie auch einen Schnauzbart haben, ist nicht eindeutig zu entscheiden. Offen muss auch bleiben, ob man den jeweils ersten Knoten zu den Enden der Armreifen hin als Hüte oder Baskenmützen ähnliche Kappen verstehen darf, wie Teržan vorgeschlagen hat. Das erscheint fraglich, wenn man den Blick auf die vergleichbaren Armreifen der venetischen Este-Kultur richtet, wie beispielsweise jenen aus Grab 8 im Gräberfeld EsteCasa Alfonsi. ${ }^{28}$

Teržan hat für die Kopfdarstellung bereits den Vergleich zu frühlatènezeitlichen Armreifen mit Pufferenden der Kelten gezogen, die zwischen dem mittleren Rheinland, der Nordschweiz und dem Dürrnberg bei Hallein

28 Chieco Bianchi, Calzavara Capuis 1985, Taf. 258/3. 

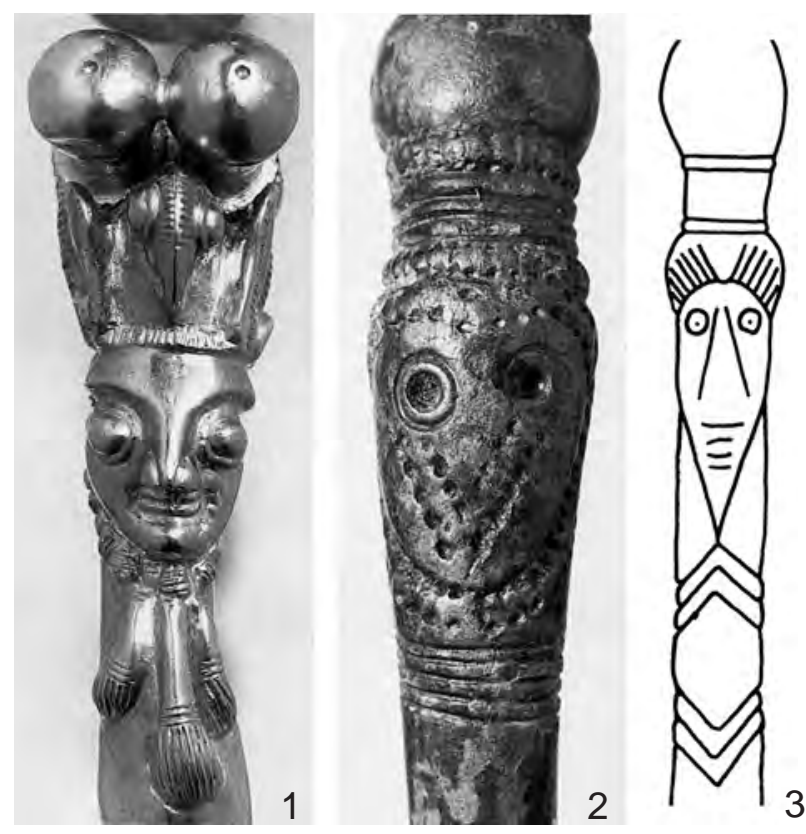

Abb. 4. Kopfdarstellungen auf frühkeltischem Ringschmuck. Ohne Maßstab. - 1. Reinheim. Gold (Krausse, Beilharz 2012, 103 und Abb. 107). - 2. Beuren, Kupp-Hügel VII/2. Bronze (CoRdiEHackenberg 1992a, 146 und Abb. 1/2). - 3. Theley, Hügel IX/2. Bronze (Cordie-Hackenberg 1992b, 174-175 und Abb. 5).

nachgewiesen und von Rudolf Echt zusammengestellt worden sind. ${ }^{29}$ Dabei favorisierte sie die Vorstellung, dass mit Blick auf den Armreif aus Novo mesto nicht der Armreif, sondern die Idee ihren Weg von den Kelten in den Südostalpenraum gefunden hat, begleitet von echten Importen, die sie als Gastgeschenke interpretiert, wobei dieser Weg auch über die venetische Este-Kultur oder über die Bernsteinstraße geführt haben kann, wie das Verbreitungsbild der durchbrochenen Gürtelhaken bzw. der Fibeln vom Typ Altmark-Kowałowice besonders eindringlich erhellt.. ${ }^{30}$

Schließlich sind aus dem nordalpinen Raum zwei Armringe mit je drei Gruppen stilisierter Köpfe zu nennen. Bis zum äußersten stilisiert erscheinen die gegenständigen, durch jeweils eine dreiteilige Knotengruppe getrennten Gesichter auf einem bronzenen Armreifen im La Tène Azeitlichen Grab von Speikern bei Nürnberg (Abb. 3/5). ${ }^{31}$ Demgegenüber sind die drei gegenständig angeordneten Kopfgruppen am goldenen Armring (Abb. 3/2, 2a) aus dem reichen Latène A-zeitlichen Grab der „Fürstin“ von Bad Dürkheim in der Pfalz (ca. 450-400 v. Chr.) in herausragender Qualität gearbeitet und lassen an der mediterranen und

29 Еснт 1999, 40-53.

30 TERŽAN im Druck.

31 Binding 1993, 90 und Abb. 34/9. damit wohl etruskischen Vorlage keinerlei Zweifel. ${ }^{32}$ Das Grab enthielt denn auch neben etruskischem Trinkgeschirr, zwei Trinkhörnern und einem zweirädrigen Wagen kostbaren Schmuck aus Gold und Bernstein. ${ }^{33}$ Der aus sechs identischen Elementen aus Goldblech zusammengesetzte Armring zeigt zwischen drei Knoten jeweils eine Gruppe aus zwei gegenständig orientierten Köpfen, die durch einen Wulst getrennt sind. Die Köpfe sind in leichter Stilisierung dennoch einigermaßen naturalistisch ausgeführt, zeigen Augen, Nase, Mund, (tiergestaltige?) Ohren und die Frisur. ${ }^{34}$ Ähnlich fand sich in dem reichen, etwas jüngeren Latène A-zeitlichen „Fürstinnengrab“ aus Reinheim im Saarland (ca. 380 v. Chr.) u. a. mediterranes Trinkgeschirr und kostbarer Goldschmuck, darunter Ringschmuck mit etruskisierenden Köpfen (Abb. 4/1). ${ }^{35}$ Aus Bronze wäre schließlich ein frühlatènezeitlicher Armreif aus La Charme aux Bois bei Troyes in den Ardennen zu nennen, den Vincent und Ruth Megaw mit Fragezeichen erst dem 3. Jh. v. Chr. (Stufe La Tène B2) zugerechnet haben, ohne dessen älteres „Formular" zu verkennen. ${ }^{36}$ Eine Datierung noch ins 4. Jh. v. Chr. dürfte zutreffender sein.

Dieses Bild, von engsten Beziehungen zu Vorlagen aus dem mediterranen und damit insbesondere etruskischen Raum verdichtet sich bei der Betrachtung von Fingerringen mit Kopf- bzw. Gesichtszier. In Litija bei Vače in Unterkrain wurden bereits im 19. Jh. in einem Grab an derselben Hand zwei massive bronzene Fingerringe gefunden; es gab keine weiteren Grabbeigaben oder Trachtelemente. An einem der beiden Ringe (Abb. 5/1) ${ }^{37}$ stoßen zwei schnauzbärtige Köpfe an einer ursprünglich mit einer Einlage aus Koralle gefüllten Fassung aneinander. Am Kinn gehen beide Köpfe in ein weiteres, jeweils anschließendes Gesicht über. Am anderen, unwesentlich kleineren Ring stoßen zwei Köpfe direkt gegenständig aneinander. ${ }^{38}$ Die Köpfe auf den beiden Fingerringen aus Vače sind der Form der Ringe angepasst und erscheinen entsprechend länglich. Sie sind zudem stark stilisiert, haben Augen in Form von Kreisaugenstempeln, eine Frisur in Wulstform mit Schrägstrichen, eine leicht plastische Nase und eine Mundritzung mit einem

32 Jолснім 1992, 182 und Abb. 1.

33 Rieckhoff, Biel 2001, 292. - Krausse, Beilharz 2012, 102-103 und Abb. 103-104.

34 Jолснім 1992, 182 und Abb. 1.

35 Еснт 1999. - Vgl. u. a. auch Rieckhoff, Biel 2001, 341-343 und Taf. 22-23. - Krausse, Beilharz 2012, 103 und Abb. 107.

36 Megaw, Megaw 1989, 135 und Abb. 206.

37 Megaw 1965-1966, 164-165/Nr. 35 und Taf. 15/4, 6. - Vgl. auch STARÈ 1953, 264 und Taf. 9/2. - Božič 1983, 105 und Abb. 46. - DuLAR 1999, 116 mit Abb.

38 Megaw 1965-1966, 164-165/Nr. 34 und Taf. 15/5-6. 

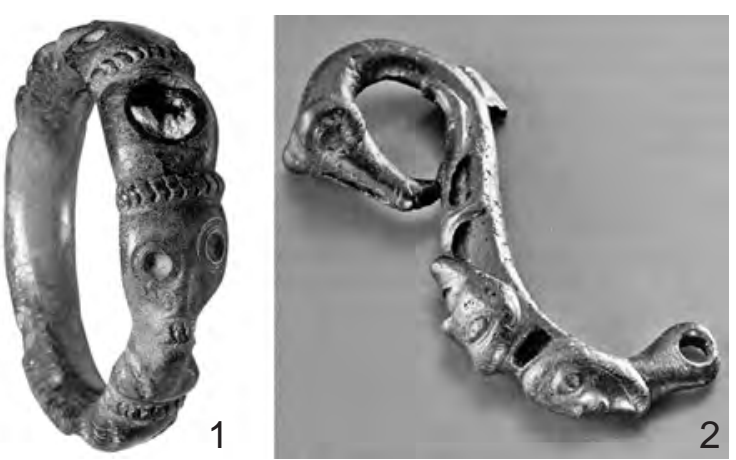

Abb. 5. Kopfdarstellungen auf einem Fingerring und einer Fibel aus dem 5. Jh. v. Chr. Bronze, Maßstab 2:1. - 1. Vače (Dular 1999, 116). - 2. Berlin-Niederschönhausen. Ohne Maßstab (Hess 2015b, 332 und Abb. 19).

durch senkrechte Kerben angezeigten Schnauzbart. Sie gelten bis heute als singulär und wurden nach der Einschätzung von V. Megaw vermutlich vom gleichen zweitklassigen regionalen Handwerker hergestellt. ${ }^{39}$ Megaw hat mit Blick auf den Ring mit der Koralleneinlage zwischen den beiden Köpfen auch bereits auf etruskische Vorlagen - er nennt goldene Fingerringe aus dem 5./4. Jh. v. Chr. aus Vulci und Bologna $a^{40}$ - hingewiesen und eine Deutung der Fingerringe als Ausdruck frühkeltischer Kunst im späthallstättischen Südostalpenraum eher bezweifelt. ${ }^{41}$

Janez Dular hat die beiden Fingerringe aus Vače zuletzt der 2. Hälfte des 5. oder dem 4. Jh. v. Chr. zugewiesen. ${ }^{42}$ Stilistisch gesehen erwog er - ebenso wie Teržan, die zugleich auf mehrere frühlatènezeitliche Gräber im Areal südöstlich um Hügel I hinweist ${ }^{43}$ - eine Inspiration seitens der frühkeltischen Kunst. Etruskische Vorbilder sind für die frühlatènezeitlichen Fingerringe bei den Kelten nicht zu übersehen. Das gilt für den goldenen Fingerring aus dem Latène A-zeitlichen „Fürstengrab“ von Rodenbach in der Pfalz (Abb. 6/2), ${ }^{44}$ ein Kriegergrab aus der Zeit um 400 v. Chr. mit goldenem Schmuck und mediterranem Trinkgeschirr ebenso wie für den etwa zeitgleichen Fingerring mit Maskenzier aus einer Siedlungsgrube am Aster Weg

39 Megaw 1965-1966, 165.

40 Megaw 1965-1966, 103 und Taf. 4/1-5.

41 Zustimmend TAppert 1994, 56-57.

42 Dular 1999, 116. - Vgl. zuerst von Hochstetter 1883, 167 und Abb. 9.

43 TeržAn 2009, 87-88.

44 Megaw 1965-1966, 101-102 und Taf. 2/1-4. - Zuletzt BlüMeL 2010, 152 mit Abb. - Zum Grab: Engels 1972.

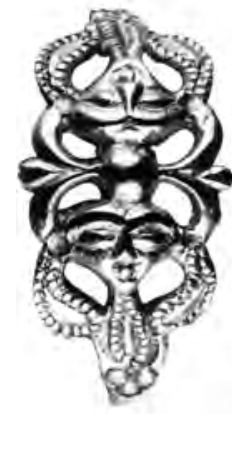

1

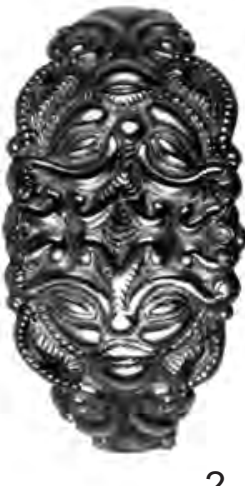

2

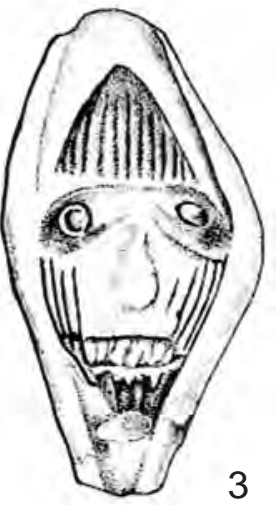

Abb. 6. Fingerringe aus dem 5. Jh. v. Chr.-1. Sardinien. Gold, Maßstab 2:1 (Megaw 1965-1966, 97, 160/Nr. 19 und Taf. 1/1-4). - 2. Rodenbach. Gold, Maßstab 2:1 (BlüMel 2010, 152). - 3. Straubing, Asterweg. Bronze, Maßstab 2:1 (TAPPERT 1994, 56-57 und Taf. 2/1).

(Lehmgrube Jungmeier, Objekt 15) in Straubing in Niederbayern (Abb. 6/3). ${ }^{45}$ Für den Fingerring aus Rodenbach hat V. Megaw auf ein Vergleichsstück aus Sardinien (Abb. 6/1) hingewiesen. ${ }^{46}$ Zwei Erklärungsmodelle stehen sich also gegenüber.

Was die Zier mit gegenständigen Köpfen bzw. Gesichtern betrifft, ist schließlich auf die Bügelzier Latène A-zeitlicher Fibeln hinzuweisen. Auf einer Maskenfibel aus Berlin-Niederschönhausen (Abb. 5/2) 47 $^{7}$ sind die beiden Köpfe länglich gehalten. Die elliptischen Augen zeigen senkrechte Pupillenschlitze, einer der Köpfe trägt einen Vollbart, der direkt in die Kurzhaarfrisur übergeht. Am Fußende der Fibel findet sich ein Widderkopf in prominenter Ausführung. Eine gleichartige Maskenfibel aus dem Umfeld der Heuneburg an der oberen Donau zeigt auch am hochgezogenen Fibelfuß einen weiteren Kopf.48 Das etwas abgenutzte Objekt lässt am Kopf am Fußende der Fibel noch einen Schnauzbart erkennen.

\section{Appliken von Köpfen bzw. Gesichtern („Masken“)}

Das Aufkommen menschlicher Figuren und Köpfe bzw. Gesichter („Masken“) geht sowohl bei den Völkern und Stämmen im Südalpenraum wie auch bei den nordalpinen Kelten auf einen mediterranen und damit insbesondere auf einen Einfluss seitens der Etrusker zurück. Besonders

45 Tappert 1994, 56-57 und Taf. 2/1. - Freundlicher Hinweis D. Božič, Ljubljana.

46 Megaw 1965-1966, 97, 160/Nr. 19 und Taf. 1/1-4.

47 Hess 2015b, 332 und Abb. 19.

48 Hess 2015b, 331-332 und Abb. 14. 
deutlich lassen sich diesbezüglich beispielsweise für Köpfe mit spitzen Ohren antike Satyrbilder als Vorbilder erkennen. ${ }^{49}$ Matthias Jung hat kürzlich gezeigt, dass etruskische Kippfiguren, an denen Floral-Ornamentales in Figürliches übergeht, was Paul Jacobstahl als Cheshire-Style bezeichnet hatte, das Vorbild der „keltischen Palmettengesichter" waren. ${ }^{50}$ Dabei bleibt es eine weitere, ebenso wesentliche Frage, inwieweit in Mitteleuropa mit den mediterranen Vorlagen auch mediterrane Inhalte rezipiert wurden, oder es zu einer „selektiven Rezeption und Transformierung ${ }^{\text {“51 }} \mathrm{kam}$, wie Hans Nortmann meint.

Das prägnanteste und - abgesehen von der Steinzeit älteste Gesicht aus dem Ostalpenraum stellt die bronzene "Maske“ aus dem Kröllkogel in Kleinklein in der Weststeiermark (Abb. 7) dar. ${ }^{52}$ Das „fürstliche“ Grab datiert in eine fortgeschrittene Phase des Horizontes Hallstatt D1 (entspricht Stufe der Schlangenfibeln), demnach in die Zeit um 580/550 v. Chr..$^{53}$ Auch wenn die „Maske“ aus Kleinklein $17,3 \mathrm{~cm}$ hoch und damit fast zwanzigmal größer ist als die Köpfe auf dem Armring aus Möllbrücke, zeigt das Gesicht aus Kleinklein in ähnlicher Weise eine durch ein Zickzackband angedeutete Frisur, leicht nach unten ziehende Augenschlitze, eine plastisch gearbeitete Nase und eine waagrechte Mundöffnung. Nach früheisenzeitlichen Vorbildern bei den Etruskern dürfte sie einst entweder auf einem hölzernen Behälter befestigt gewesen sein, der den Leichenbrand enthielt, oder zusammen mit den beiden linken Händen eine hölzerne Stele geziert haben, die den Toten darstellen sollte; eine Deutung, die Markus Egg und Jasmin Munir zuletzt favorisiert haben. Die technischen Merkmale lassen keinerlei Zweifel daran, dass die "Maske“ und die Hände nicht in Etrurien, sondern vor Ort hergestellt wurden. Es ist längst bekannt, dass im Südostalpenraum wie auch bei den Rätern in Alt-Tirol während der Hallstattkultur reichlich Fremdes kopiert bzw. in lokalem Kolorit hergestellt wurde. ${ }^{54}$

Blickt man nach Etrurien, erinnert die Ausführung der Gesichter am Armreif aus Möllbrücke, vor allem was die Umsetzung von Frisur, Nase und Mund betrifft, zudem an ein Köpfchen auf einem goldenen Diadem aus dem Arsenale Militare in Bologna (Abb. 8/1). ${ }^{55}$ Dem könnte

49 Jacobsthal 1944, 21. - Megaw 1965-1966. - Von Hase 1973. FREY 2002b, 202.

50 JUNG 2008.

51 Nortmann 2016, 222-223.

52 EGG 2013, 466-467. - EGG, Munir 2013, 166-174.

53 Teržan, Črešnar 2014, 719-724.

54 Siegfried-Weiss 1979, 126-130. - EgG 1996, 265-273 und Abb. 146-150. - Für Slowenien: Gabrovec 1992. - Für das Rätergebiet: GleirSCHER 1993a. - GleIrSCHER 1993b.

55 Von Hase 1973, 55 und Taf. 4/ 4.

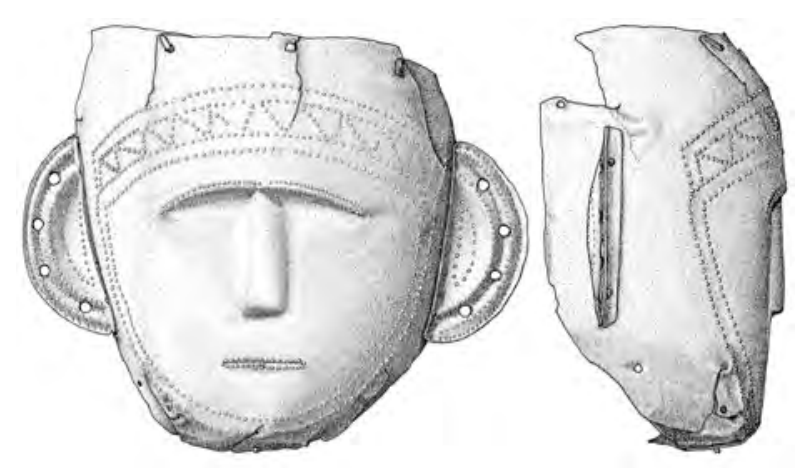

Abb. 7. Kleinklein, Kröllkogel, „Totenmaske“. Bronze, Maßstab 1:4 (EGG 2013, 466-467).
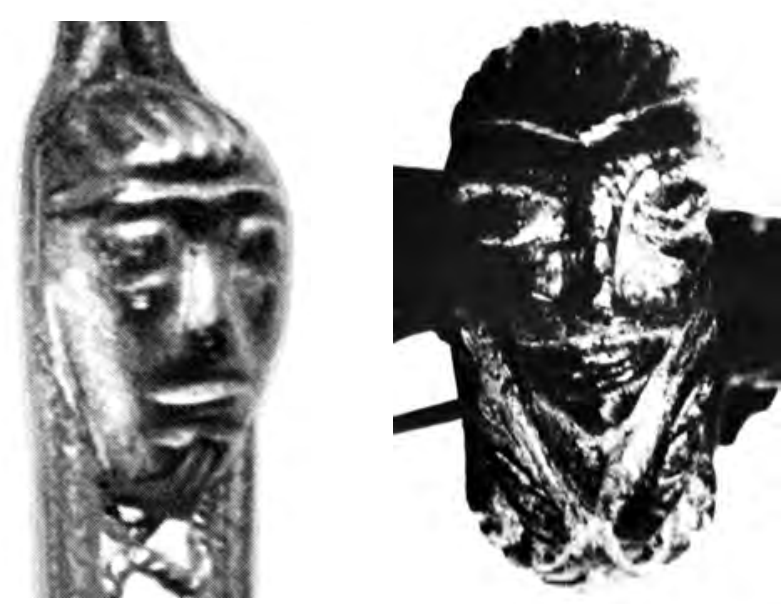

1

2

Abb. 8. Köpfe auf Kleinkunst aus Etrurien. - 1. Detail eines goldenen Diadems aus Bologna, Arsenale militare. Ohne Maßstab (Von Hase 1973, 55 und Taf. 4/4). - 2. Detail eines bronzenen Gürtelhakens ohne Fundort, Museum Florenz. Ohne Maßstab (Von Hase 1973, 59 und Taf. 8/1).

man den Kopf auf einem bronzenen Gürtelhaken aus Etrurien $(\mathrm{Abb} .8 / 2)^{56}$ ebenso anschließen wie die etwas naturalistischere Ausführung auf einem Anhänger aus Goldblech aus dem Gräberfeld Bucacce in Bisenzio. ${ }^{57}$ Sie alle haben allerdings in naturalistischer Sichtweise hervorquellende, linsenförmige Augen. Wie wiederholt festgestellt wurde, ergibt sich daraus insofern ein gewisses Problem, als die etruskischen Vergleichsstücke ins 7. bis frühe 6. Jh. v. Chr. datieren, demnach um gut hundert Jahre zu alt sind, um als

56 Von Hase 1973, 59 und Taf. 8/1.

57 Von Hase 1973, 55 und Abb. 1. 

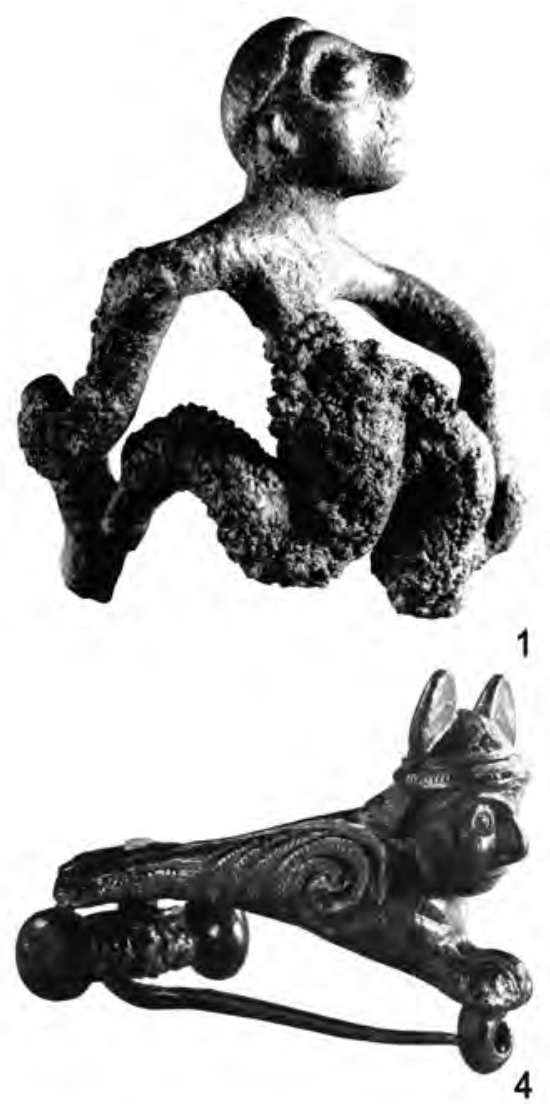

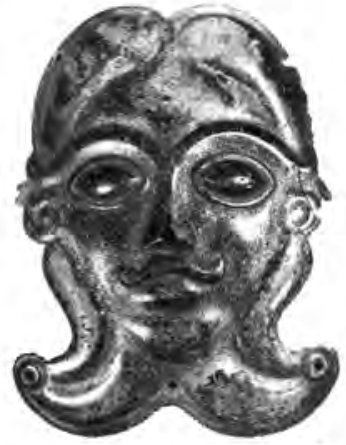

2

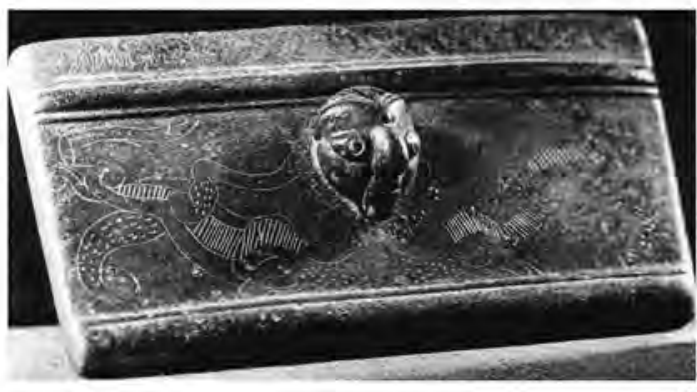

3

Abb. 9. Köpfe in der Kleinkunst der frühen Kelten. Bronze. - 1. Völkermarkt, Führholz. Maßstab 2:1 (Wedenig 1990, 192 und Abb. 29-30). 2. Hallein, Dürrnberg. Maßstab 1:2 (Penninger 1972, 78/Nr. 3 und Taf. 42/A3). - 3. Stupava. Maßstab 1:1 (Megaw 2010, 611 und Abb. 1-2). - 4. Ossarn. Maßstab 2:1 (Megaw 2010, 616 und Abb. 15a). - 5. Rascheid. Ohne Maßstab (Haffner 1992, 157/Nr. 42). - 6. Thomm. Ohne Maßstab (Hafrner 1992, 157/Nr. 44). - 7. Schwieberdingen. Ohne Maßstab (Binding 1993, 54-55 und Abb. 17/2).

unmittelbare Vorbilder für die Köpfe bzw. Gesichter auf dem Ringschmuck der Kelten oder im Südostalpenraum gelten zu können. Friedrich-Wilhelm von Hase meinte, dass entsprechende Importstücke nördlich der Alpen bis ins 5. Jh. v. Chr. im Umlauf gewesen sein müssten und spät, aber doch, die Ausbildung des frühkeltischen Stiles nachhaltig beeinflusst hätten. ${ }^{58}$ Besteht tatsächlich ein Hiatus, ist an eine regionale Neuschöpfung des Bildtypus zu denken.

FürdieDarstellung eines Kopfes, dieHaare, Augen, Nase und Mund hervorhebt, finden sich im Bereich der frühkeltischen Kunst zahlreiche Belege, etwa der von Ulrike Binding als naturalistisch eingestufte Kopf auf der Maskenfibel aus Schwieberdingen bei Stuttgart (Abb. 9/7). ${ }^{59}$ Unter den Kopfappliken auf frühlatènezeitlichen Gürtelhaken könnte man als Beispiel das Latène A-zeitliche Exemplar von Stupava unweit Bratislava (Abb. 9/3) heranziehen, mit durch einen Kreisstempel gefertigten Pupillen und im Vergleich

58 VON HASE 1973, 60.

59 Binding 1993, 87 und Abb. 33/1 sowie Kat.-Nr. 174a mit Taf. 4/4. zu Möllbrücke deutlich plastischerer Gesamtausführung. ${ }^{60}$ Pupillen in Form von Kreisaugenstempeln - und keine linsenförmigen, hervorquellenden Augen - finden sich auch an den beiden Köpfen einer Fibel aus Parsberg in der Oberpfalz, ${ }^{61}$ für die zur Diskussion steht, ob ein Mensch oder ein Tier (Fabelwesen) dargestellt ist, sowie auf einer Maskenfibel aus einem Mädchengrab aus der Zeit um 400 v. Chr. in Ossarn in Niederösterreich (Abb. 9/4), ${ }^{62}$ für die diskutiert wird, ob der Kopfaufsatz als Tierohren ( „behelmter Vogelmann“ nach Megaw) oder als Mistelkrone (nach Frey) zu deuten ist. In der frühkeltischen Kunst können die Augen sowohl linsenförmig und hervorquellend, wie auch in Etrurien, oder in hallstättischer Tradition auf

60 Megaw 2010, 611 und Abb. 1-2.

61 Binding 1993, 90 und Kat.-Nr. 143 mit Taf. 4/2. - Hess 2015a, 156. - Detailfoto u. a. bei FreY 1993, 156-157 und Abb. 118. - FreY 2002a, 178 und Abb. 143. - Frey 2002b, 201. - Megaw 2010, 611 und Abb. 6.

62 Frey 1996, 206-207 und Abb. 9/1 (Grab 17). - Vgl. auch Megaw 2010, 616 und Abb. 15a. 
Kreisaugenstempel reduziert ausgeführt sein. Das „Hallstättische“ illustriert in diesem Zusammenhang am besten der Vergleich der Augen der beiden süditalischen Löwen und des vor Ort nachgemachten Löwen auf dem Kessel aus dem Fürstengrab von Hochdorf. ${ }^{63}$

Dem sind mit Blick auf den Armreifen aus Möllbrücke stilisierte menschliche Köpfe auf frühlatènezeitlichen Anhängern aus dem südostalpinen Raum anzuschließen, mit einer Frisur aus seichten Kerben, einer leicht herausgearbeiteten Nase, einem eingeritzten Mund und Augen in Form von Kreisaugenstempeln (Abb. 10/4). ${ }^{64}$ Bereits Gero von Merhart hat halbplastische Bronzefigürchen aus dem mittleren Alpenraum (Abb. 11/1) erörtert, darunter Adoranten. ${ }^{65}$ Ihre Ausführung ist auf das Wesentlichste reduziert und einigermaßen stilisiert. Die hier interessierenden Köpfe sind durch Augen in Form von Kreisaugenstempeln, eine plastische Nase und einen Mundschlitz sowie seitlich und anatomisch beliebig angesetzte Ohren gekennzeichnet. Von Merhart erkannte die Figürchen als heimische Produkte des 5. und 4. Jhs. v. Chr. und leitete die Motive - Adoranten und Caestuskämpfer - von Einflüssen aus der venetischen Kultur ab. Mit entsprechenden Veränderungen im Kult sind sie als mediterran-etruskischer Einfluss einzuschätzen. ${ }^{66}$ Ein ähnliches, plastisch gefertigtes Figürchen stammt aus Hügel I/Grab 2 in Stična in Slowenien, auch wenn man über den Grabverband wird diskutieren müssen. ${ }^{67}$ Die Frisur wird wie bei der „Maske“ aus Kleinklein (Abb. 7) durch ein Zickzackband hervorgehoben - wenn damit nicht eine Kopfzier gemeint ist -, die Augen sind lochförmig, der Mund als Schlitz gestaltet, die Nase wenig profiliert. Hier zeigt sich, wie beispielsweise auch in der Situlenkunst, eine parallele Entwicklung vom mittleren Alpenraum bis in den Südostalpenraum.

Grobschlächtige Appliken menschlicher Köpfe mit Augen in Form von Kreis(augen)stempeln, plastischen Nasen und Mündern, die wiederholt Zähne erkennen lassen, prägen das Erscheinungsbild frühlatènezeitlicher bronzener Anhänger im Bereich der rätischen Fritzens-SanzenoKultur (Abb. 10/1). Während ich am Beginn einer zuletzt

63 Biel 1985, 119-129. - Gauer 1985, 126-129. - Megaw, Megaw 1989, 42 und Abb. 35-36.

64 Corazza, Vitri 2001, 48-56 und Abb. 58 (Paularo, Grab 18), 65 (Vače), 66 (Sv. Lucija). - Gleirscher 2018a, 66-67.

65 Von Merhart 1932. - Ergänzungen bei Kromer 1974. - Wells 1978. - ViTRI 1997, 582/Nr. 27 und Abb. 14/2. - Deutlich reduzierter und von anderem Typ, mitunter mit Augen aus Kreisaugenstempeln, sind mehrere bronzene Figürchen aus Šmarjeta: Dular 1980, Abb. 11/4. - Vgl. dazu SzILÁgyi 1992, 223-224 und Abb. 1-2.

66 Gleirscher 1993b, 83-85 und Abb. 11-12; 91 und Abb. 22.

67 Wells 1981, 48 und Abb. 29/g-h. - Vgl. bereits Wells 1978.-Zur Problematik der Grabinventare der Sammlung Mecklenburg: WeIss 1993. - Božıč 2009. - BoŽIČ 2010. regen Diskussion eine Deutung als Raitia-Bilder und damit einen Bezug zu einer allumfassenden, der griechischen Artemis vergleichbaren Mutter- und Stammesgottheit vorgeschlagen habe, ${ }^{68}$ sprach Markus Egg ${ }^{69}$ zeitgleich und neutraler von einer „Herrin der Pferde“. ${ }^{70}$ Für eine vergleichbare Kopfapplike auf einer Halbmondfibel aus Völs am Schlern in Südtirol (Abb. 10/2) hat Lorenzo Dal Ri eine Interpretation in Form einer stark stilisierten geflügelten Figur vorgeschlagen, die Kopfappliken mit den deutlich kenntlichen Zähnen mit einem Gorgoneion verglichen. ${ }^{71}$

Mit dem Motiv „Herrin/Herr der Tiere“, der Potnia Theron bzw. des Despotes Theron haben sich letzthin Bettina Arnold und Derek B. Counts ausführlich beschäftigt und dazu auch einen Sammelband herausgegeben. ${ }^{72}$ Sie führen das Motiv des Mischwesens aus menschlichen und tierischen Elementen bis ins Paläolithikum zurück. Über den Vorderen Orient gelangte das Motiv bekanntlich nach Griechenland, wo die weibliche Variante in Form der archaischen Artemis eine dominante Stellung einnimmt, und weiter nach Italien, wo Löwen und Vögel als Begleittiere überwiegen, die weibliche Figur wiederholt geflügelt erscheint und damit auch selbst zum Mischwesen wurde. Die mediterranen Vorbilder datieren ins 7./6. Jh. v. Chr., woraus sich mit Blick auf die Etrusker eine Art Lücke zur Entstehung der Latènekultur um die Mitte des 5. Jhs. v. Chr. ergibt, nicht aber mit Blick auf den zirkum- und inneralpinen Raum.

Jennifer M. Bagley hat sich in diesem Zusammenhang ausführlich mit den frühlatènezeitlichen durchbrochenen Gürtelhaken der Variante Castaneda beschäftigt, die im zirkumalpinen Raum beheimatet sind. ${ }^{73}$ Am Paradebeispiel aus Hölzelsau bei Kufstein sind die Augen der Pferdeköpfe in Form von Kreisaugenstempeln gemacht, also nicht linsenförmig keltisch. Was die Vergleichbarkeit der „Herrin der Tiere“ bei den Kelten nordwärts der Alpen und einer

68 Gleirscher 1986. - Mit Vertiefung: Gleirscher 2002a, 205-206 und Taf. 147/A. - Gleirscher 2002b, 614-618. - Vgl. auch GleirSCHER 2009, 37-66. - Gleirscher 2011, 123-132. - Weiters TeržAN $1990,86-87$.

69 EgG 1986a. - Vgl. auch Gamper 2006, 368-369: Göttin, die zwei Pferde am Halfter oder am Zügel hinter sich herführt.

70 Grundsätzlich zustimmend und wiederholt als „Herr/Herrin der Tiere“ angesprochen, u. a. bei: TeržAn 1990, 88. - METZnER-NebeLSICK, NeBELSICK 1999, 80-82, 93-99. - KossaCK 1993, 150.- KosSACK 2002, 312. - MARzATICO 2002. - MARZATICo 2012, 320-322. - Rossi 2005, 26. - Appler 2006, 14. - LANG 2010, 21-22. - DAL Ri 2012, 153-156. - Ablehnend Tomedi 2002, 1226-1227, 1230.

71 DAL Ri 2012, 149-153, 157. - Vgl. zur Fibel auch TERŽAN 1990, 88 und Abb. 16.

72 Counts, Arnold 2010. - Vgl. zusammenfassend auch Bagley 2013, 70-72. - BAGLEY 2014, 210-239.

73 Bagley 2013. 

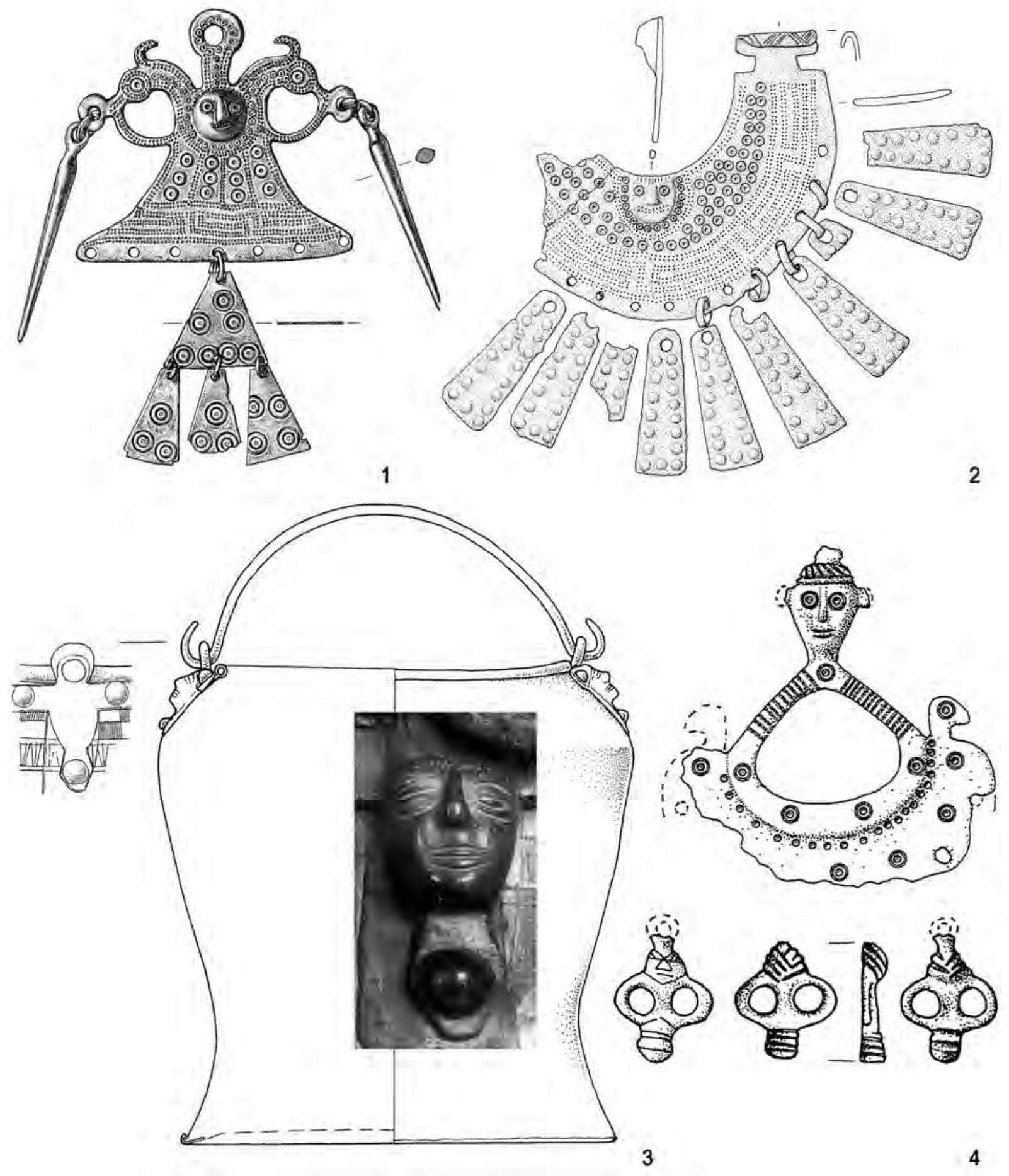

Abb. 10. Gesichtsappliken aus dem mittleren Südalpenraum, 5./4. Jh. v. Chr. Bronze. - 1. Fundort unbekannt, wohl Nonsberg. Maßstab 1:2 (EGg 1986a). - 2. Völs am Schlern, Peterbichl. Maßstab 1:2 (DAL Ri 2012, 149-153, 157). - 3. Valle di Cadore, fondo Chiamulera. Maßstab 1:3 (Calzavara 1984, 866). - 4. Paularo, Misincinis. Maßstab 1:1 (Corazza, Vitri 2001, 48-56 und Abb. 58). 

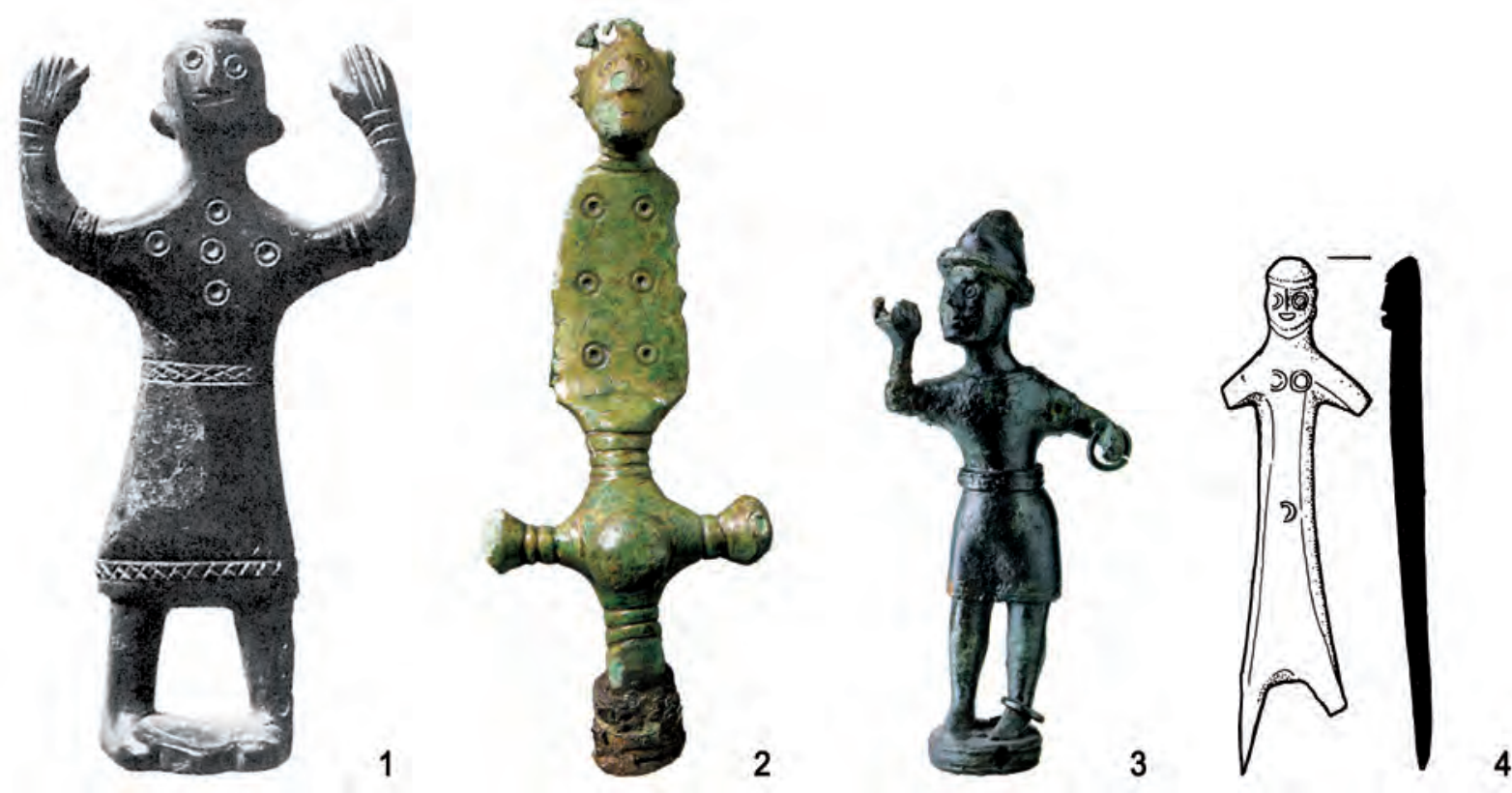

Abb. 11. Figürchen und Gesichtsapplik aus dem Südostalpenraum, 5./4. Jh. v. Chr. Bronze. - 1. Gramais, Parzinspitze. Maßstab 1:1 (Von Merhart 1932, 57 und Abb. 1). - 2. Vinica. Maßstab 1:1 (Božıč 1999b, 179). - 3. Idrija pri Baca. Maßstab 1:2 (Božı̌ 1999a, 176). - 4. Šmarjeta. Maßstab 1:1 (Dular 1980, Abb. 11/4).

weiblichen Muttergottheit bei den inneralpinen, von den Etruskern stark beeinflussten Kulturgruppen anbelangt, wurde wiederholt auf die Nähe zur griechischen Artemis hingewiesen. ${ }^{74}$ So erkennt Rudolf Echt das Motiv in seiner weiblichen und männlichen Ausprägung am Armring aus dem Grab der Fürstin von Reinheim, an dem beide Elemente kombiniert und demnach eigenen Vorstellungen angepasst erscheinen..$^{75}$

Dem ist die bereits erwähnte bronzene Statuette des Hornbläsers aus Idrija bei Bača unweit von Tolmin im Isonzotal/Sočatal (Abb. 11/3) anzuschließen. Sie wurde 1887 in einem Brandgrab gefunden und dank des Negauer Helmes dem 5. (Egg) bzw. 4. (Guštin und Božič) Jh. v. Chr. zugewiesen. ${ }^{76}$ Das naturalistische Gesicht erfährt durch die in Form von Kreisaugenstempeln gefertigten Augen einen besonders eigentümlichen „südostalpinen“ Charakter, wird zur Mischung aus mediterraner Plastik und stilisierter, südostalpiner Darstellungsweise. In unserem Zusammenhang

74 Kelten: Eсht 1999, 45. - BAgLey 2013, 74-75. - Räter: GleiRSCHER 1986. - GLEIRSCHER 2002a, 206-207. - GLEIRSCHER 2002b, 616-617. - Noriker: Gleirscher 2009, 37-38. - Gleirscher 2011, 125-126. - TeržAn 2011. - In diesem Sinn u. a. auch Guggisberg 2000, 277. - Vgl. weiters Guggisberg 2010.

75 EсHт 1999, 253-270. - Zustimmend Guggisberg 2000, 256-262 oder BAgLeY 2013, 75.

76 GušTin 1980. - Egg 1986b, 114-115 und Abb. 53/2; Taf. 290/b.Beste Abbildung bei Božıč 1999b, 179. ist zudem auf den Arm- und Fußring der Statuette hinzuweisen, beide linksseitig getragen. Das entspricht Beobachtungen zu Männergräbern in Unterkrain, wobei für diese Armringe Legierungen aus Blei und Zinn charakteristisch sind. Dem entspricht die Umsetzung der Augen am Kopf auf einer Dreiknopffibel aus dem 4. Jh. v. Chr. aus Vinica (Abb. 11/2). ${ }^{77}$ Ihre Fußbildung erinnert noch an frühe Dreiknopffibeln der Picenter aus der 2. Hälfte des 6. Jhs. v. Chr. ${ }^{78}$

Auf einer bronzenen Situla aus dem Cadore im Veneto (Abb. 10/3), die einem südalpinen Situlentyp des 4. Jhs. v. Chr. zuzuordnen ist, tragen die Attaschen eine plastische Kopfzier. ${ }^{79}$ Während die Kopfappliken mit keltischem anstelle von etruskisch-italischem Einfluss erklärt werden, ${ }^{80}$ lassen sich für muschelförmig gestaltete Attaschen an gleichartigen Situlen zweifelsfrei etruskische Vorbilder ausmachen. ${ }^{81}$ Das wird man deshalb auch für die Kopfappliken erwägen müssen, insbesondere mit Blick auf eine Attaschenzier auf einem Eimer aus einem Grab in Bologna/ Arnoaldi. ${ }^{82}$ Aus Padua und aus Mel unweit von Belluno

77 Gabrovec 1966, 70 und Taf. 14/1. - Božıč 1999a, 176.

78 Seidl 2005, 58 und Abb. 1/ 4. - Preložnik 2007, 124 und Abb. 1.

79 Calzavara 1984, 866.

80 Ruta SERAFini 1998, 52 spricht von einer keltischen Sitte im Zusammenhang mit Kopftrophäen. - TuRk et al. 2009, 53-54 und Abb. 6.

81 Turk et al. 2009, 53-54 und Abb. 7-9.

82 Macellari 2003, 95 und Taf. 7/1; 85. 
sind Kopfappliken aus Bronzeblech bekannt geworden, die ein Gesicht in naturalistischer Ausführung zeigen, mit ovalen Augen samt Augenbrauen sowie plastisch modellierter Nase und Mund. ${ }^{83}$ Sie werden dem 2./1. Jh. v. Chr. zugeordnet und sind demnach deutlich jünger als die Köpfe auf dem Armreif von Möllbrücke. Späthallstattzeitlich sind hingegen die in Reihen gegenständig angeordneten Köpfe auf bronzenen Blechen von Zeptern aus den Gräbern 10 und 11 in Alpago-Pian de la Gnela unweit von Belluno. ${ }^{84}$

Unzweifelhaft der frühkeltischen Kleinplastik zuzuordnen ist hingegen ein 2,9 cm hohes Figürchen, das in Führholz bei Völkermarkt (Abb. 9/1) in einem von der beginnenden Eisenzeit bis gegen 300 v. Chr. belegten Hügelgräberfeld gefunden wurde. ${ }^{85}$ Das Kriegergrab der Stufe Latène A (ca. 450-380 v. Chr.) ist nach Einschätzung von Reinhold Wedenig ein Flachgrab, wahrscheinlicher aber ein verschliffenes Hügelgrab. Die Ausstattung umfasst u. a. eine kleine Bronzeplastik, deren funktionale und ikonografische Ansprache bis heute nicht überzeugend geglückt ist ${ }^{86}$ weil das Objekt stark unter dem Feuer des Scheiterhaufens gelitten hat. ${ }^{87} \mathrm{Die}$ 2,9 cm hohe bronzene Figur lässt jedenfalls einen Mann im Knielaufschema erkennen, dessen Gesicht unzweifelhaft Züge keltischen Kunsthandwerks zeigt (vgl. Abb. 9/2-3, 5-7). ${ }^{88} \mathrm{Zu}$ nennen sind insbesondere die hervorquellenden linsenförmigen Augen sowie der durch Einstiche wiedergegebene Schnauzbart. ${ }^{89}$ In Führholz ließen sich auch eine Reihe weiterer frühlatènezeitlicher Objekte in späthallstattzeitlichem Kontext nachweisen, etwa eine Latène B1zeitliche Fibel und ein durchbrochener Latène A-zeitlicher Gürtelhaken im Umfeld von Hügel 77. ${ }^{\circ 0}$ Aus Kärnten ist

83 Calzavara 1984, 855.

84 Gangemi 2015, 176 und Abb. 1.

85 Wedenig 1990, 184-193.

86 Herr der Tiere: Guggisberg, Stöllner 1996. - Stöllner 2010, 301 und Anm. 54. - Zustimmend BAgLey 2013, 70. - Skythischer Krieger: TERŽAN 1998, 532-533.

87 Vgl. zu Fragen der Rekonstruktion auch Fürhacker, PfeiferSCHÄller, WEDENig 2004, 110-111 und 115-116.

88 HaffNer 1992, 157/Nr. 42 (Rascheid), 44 (Thomm).

89 Wedenig 1990, 192 und Abb. 29-30. - Wedenig 1994, 6 (Detailzeichnung zum Kopf). - WEDENIG 1999, 15-16 und Abb. 12. Wedenig 2005, 30 und Abb. 26.

90 Wedenig 1997, 76 und Taf. 5/7-8. - Wedenig 2005, 24 und Abb. 12/oben. - Gleirscher 2001, 212-213 und Abb. 3. - Zur Belegungszeit: Wedenig 1999, 5-6 und Abb. 4. - Wedenig 2005, 20-23 und Abb. 5. - Vgl. zu frühkeltischen Funden in späthallstättischem Ambiente in der Steiermark: Tiefengraber 2015, 596-603 (allerdings auch mit einer Reihe von späthallstättischen Fibeln mit italischen Wurzeln). - Für Slowenien vgl. zuletzt TeržAn 2009, 86 und Anm. 1-3. - TerŽAn im Druck. - Für Friaul: Vitri 2001, 27-30. - Corazza, Vitri 2001, 58-66 und Abb. 71 (Paularo, Grab 34) .Oriolo et al. 2015, 32-47. dem von der Gurina bei Dellach im Gailtal ein Latène Azeitlicher Armreif anzuschließen. ${ }^{91}$

Aus dem keltischen Kulturkreis könnte man dem Gesicht des Männchens aus Führholz den Kopf auf dem Gürtelhaken aus Grab 229/1 vom Dürrnberg bei Hallein anschließen, mit längsovalen hervorquellenden Augen. ${ }^{92}$ Demgegenüber könnte man den kleinen, etwas grobschlächtigen Kopf an einem Fingernagelreiniger aus dem La Tène B1-zeitlichen Grab 44/1 vom Moserstein am Dürrnberg bei Hallein (Abb. 12/1) wiederum als Vergleich zu den Köpfen am Armreif von Möllbrücke nennen; ${ }^{93}$ dieser ist aber in stilistischer Hinsicht ob der Kleinheit nicht überzubewerten. Ludwig Pauli sah in der Maskenverzierung eine Bestätigung für die Integration des hallstättischen Toilettegerätes in die Latènekultur. ${ }^{94}$ Inzwischen sind aus dem östlichen Oberitalien eine Reihe vergleichbarer Fingernagelschneider (Abb. 12/2-3) - von den Bearbeitern als „lanzettförmige Anhänger“ eingestuft - aus dem 5. Jh. v. Chr. bekannt geworden, in zwei Fällen mit gegenständig verdoppeltem Kopfaufsatz, wobei die Stilisierung der Gesichter vor allem an einem Exemplar aus Este wiederum den Gesichtern am Armreif aus Möllbrücke anzuschließen ist. ${ }^{95}$

Für den Armring aus Möllbrücke stellt sich wie für die anderen, mit gegenständigen Köpfen bzw. Gesichtern verzierten Fuß-, Arm- und Fingerringe aus dem Südostalpenraum somit zunächst die Frage, inwieweit es sich dabei weniger um einen kulturellen Einfluss aus der frühkeltischen Kultur nördlich der Alpen, wie gemeinhin angenommen wird, als vielmehr um eine direkte Rezeption etruskischer Vorlagen handeln könnte, wie das V. Megaw im Gegensatz zur slowenischen Forschung mit Blick auf die beiden Fingerringe aus Vače vorgeschlagen hat. Sowohl die Darstellung der Gesichter wie auch Fingerringe waren im Südostalpenraum fremd. ${ }^{96}$ Stilistisch gesehen mögen die Gesichter auf dem Armreif aus Möllbrücke einen direkten Einfluss aus Italien auf die Kulturentwicklung in den südalpinen Tälern untermauern, der im ausgehenden 6. Jh. einsetzte und im 5. Jh. v. Chr. besonders intensiv war, wie gezeigt wurde.

91 Jablonka 2001, 132 und Taf. 92/19. - Vgl. Pauli 1978, 165-168. 92 Megaw, Megaw, Neugebauer 1989, 495 und Abb. 7/3.

93 Penninger 1972, 78/Nr. 3 und Taf. 42/A3. - Gleirscher 2018a, 62 und Abb. 2/4; Abb. 3. - Foto: Moser 2010, 76/unten. - Vgl. auch Crummy 2001

94 PAUli 1978, 260-261.

95 Nascimbene 2015, 169 und Abb. 2. - Voltolini 2015, 172 und Abb. 1. - Gleirscher 2018a, 65-66 und Abb. 6-7.

96 Worauf Biba Teržan hinweist. 


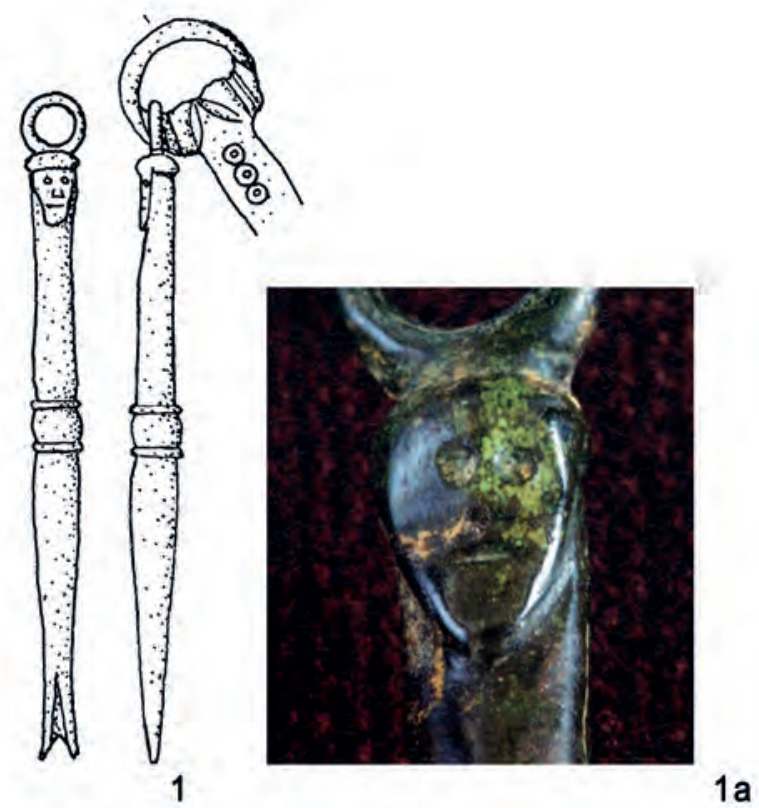

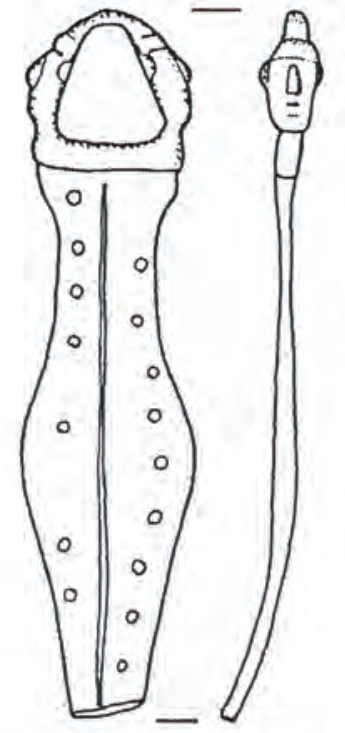

2

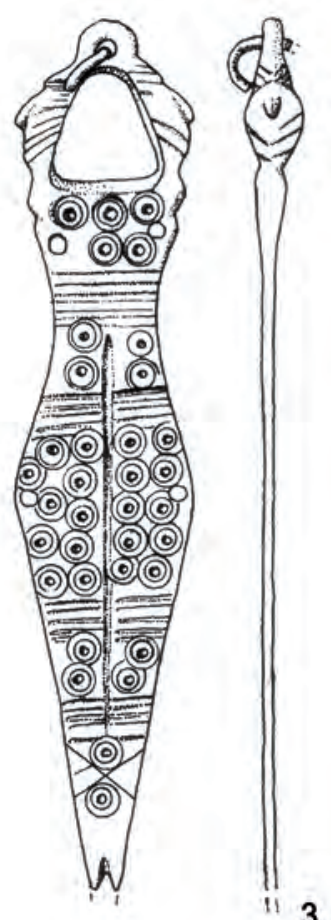

3

Abb. 12. Köpfe auf frühlatènezeitlichen Fingernagelreinigern. Bronze, Maßstab 1:1. - 1. Dürrnberg bei Hallein (PenNINGER 1972, 78/ Nr. 3 und Taf. 42/A3). - 1a. Dürrnberg bei Hallein (Foto: Moser 2010, 76/unten, Detail). - 2. Este (Voltolini 2015, 172 und Abb. 1). 3. Pieve d'Alpago, Pian de la Gnela (Voltolini 2015, 172 und Abb. 1).

\section{Zur Deutung der Köpfe bzw. Gesichter („Masken“)}

Es bleibt die wesentlich schwierigere Frage nach der Deutung der Köpfe bzw. Gesichter („Masken“). Menschliche und tierische Köpfe, oft als Mischwesen bzw. Phantasiegeschöpfe kombiniert, zählen zu den Merkmalen frühkeltischen Kunsthandwerks. ${ }^{97}$ Sie finden sich einzeln oder vervielfacht vor allem auf Fibeln und Gürtelhaken, aber auch auf Ringschmuck, Waffen und Trinkgeschirr. Hinter diesen Köpfen bzw. Gesichtern wurde zum einen ein apotropäischer Sinn vermutet, das Bild mit einem Abwehrzauber verbunden. ${ }^{98}$ Georg Kossack verstand die frühkeltischen „Masken“ in Verbindung mit Tierfiguren oder Teilen von deren Leibern zum anderen und mit Blick nach Mittelitalien als Gestalten dämonischen Charakters oder aus dem Mythos. ${ }^{99}$ In der Darstellung erkannte er "den Grundgedanken der Metamorphose menschlichen Seins, den Glauben an die Lösbarkeit und die Verwandlung der Seele (alter ego)

97 Z. B. Jacobsthal 1944. - Megaw, Megaw 1989, zu Menschenköpfen bes. 89-75. - Weiters FreY 2002b, 197-205. - FreY 2007b.

- Vgl. zuletzt u. a. Egri 2014, 79-83. - BAgLey 2014. - NorTMANN 2016, 213-218. - Unter psychologischen und ethnografischen Aspekten: Wells 2012. - Hess 2015a. - LAmb 2019, 87-91.

98 Megaw 1970, 38. - Pauli 1975, 205. - Binding 1993, 123.

99 Kossack 1993, 151-152. oder an die Epiphanie der Gottheit in Tiergestalt, die hier als Mischwesen differente Eigenschaften in sich vereinigt. “100 Dabei folge die nunmehr spiegelbildliche Wiedergabe des seit der Hallstattzeit bekannten Motivs (Vogelpferd etc.) mediterranem Einfluss.

Otto-Herman Frey wiederum, der sich wiederholt mit der Kunst der Kelten, ihrer Entwicklung und Fragen zu ihrem Inhalt beschäftigte, ${ }^{101}$ sprach von menschenköpfigen Fabelwesen, ${ }^{102}$ wies aber zugleich darauf hin, dass uns die Deutung der Geisteswelt der frühen Kelten im Einzelnen weitgehend verschlossen bleiben werde; der Bogen reiche von der Darstellung realer Menschen und Tiere bis hin zu Dämonen und Göttern. ${ }^{103}$ Eine Deutung der menschlichen Köpfe innerhalb der frühkeltischen Kunst hätte im Rahmen von pars pro toto-Vorstellungen zu erfolgen. Hinter den Köpfen sah Frey wohl anthropomorph gedachte Götter, hinter den Mischwesen Dämonen, apotropäische Gottheiten oder regionale Heroen, die von mystischen Ungeheuern

100 Kossack 1993, 152.

101 U. a. FREY 1993. - Frey 2002b. - Frey 2007a. - Frey 2007b.

102 Frey 2002b, 202.

103 Frey 2002b, 204. 
beschützt werden. ${ }^{104}$ Zudem wies er drauf hin, dass menschliche Köpfe bei den Kelten das Zentrum der Lebenskraft symbolisieren. Kommen dazu Insignien, sind darin magische Wesen zu vermuten. Wesentlich bleibe dabei, dass die Kelten trotz des Rückgriffs auf Stilmittel der etruskischen Kunst stets Symbole aus ihrer eigenen Welt wiedergaben. ${ }^{105}$

In der auch in diesem Zusammenhang relevanten Frage nach der Erscheinungsform keltischer Götter in vorrömischer Zeit gelangte Frey zu einem differenzierten Bild. ${ }^{106}$ Während es seiner Einschätzung nach in spätkeltischer Zeit zweifellos auch anthropomorphe Götterbilder gab, ${ }^{107}$ sowohl in der Großplastik wie auch in der Kleinkunst, auBerdem durch Berichte antiker Autoren belegt, bleibe deren Nachweis für die ältere Latènezeit schwieriger. Die Großplastik des ausgehenden 6. und 5. Jhs. v. Chr. lässt auch ihn unzweifelhaft einen Ahnenkult für Anführer erkennen, ${ }^{108}$ doch sind bereits für die Frühlatènezeit zudem Götterbilder zu vermuten, etwa in der janusartigen Stele von Holzgerlingen in Württemberg. ${ }^{109}$ Und noch deutlicher lasse die frühkeltische Kleinkunst göttliche und dämonische Wesen erkennen, etwa in geflügelten Figuren als „Herren/Herrinnen der Tiere/Dämonen“, vielleicht auch auf Köpfe bzw. Gesichter reduziert, die sich bis in spätkeltische Zeit finden. ${ }^{110}$ Brennus, so wird überliefert, wäre 279v. Chr. beim Betreten eines Tempels in Gelächter ausgebrochen, als er sah, dass die Griechen anthropomorphe Götterbilder fertigten (Diodor 22, 9, 4) - eine literarische Bestätigung chiffrierter Gottesbilder der Kelten während der Früh- und Mittellatènezeit, wie sie in der keltischen Kleinkunst begegnen? ${ }^{111}$

Die Zusammenschau der figürlich verzierten, frühlatènezeitlichen Fibeln durch Binding hat gezeigt, dass für die Deutung von Köpfen (bzw. Gesichtern oder „Masken“) nach wie vor zwei Muster zu verfolgen sind. Denkt man nicht an inhaltslose Variationen der Kunsthandwerker, könnten einerseits - und darauf weisen „Kronen“ aus Mistelblättern (Abb. 9/2, 4-6) - Bilder von Göttern ${ }^{112}$ oder von vergöttlichten Herrschern bzw. Heroen gemeint sein, andererseits Köpfe von im Kampf getöteten Kriegern, têtes

104 Frey 1993, 157. - Frey 2002b, 202-203. - Vgl. auch MEgAw 2010, 617-618, mit Literatur.

105 FREY 1993, 160-161.

106 FREY 2007b.

107 FREY 2007b, 203-204, 207.

108 FREY 2007b, 204-205.

109 Frey 2007b, 205. - Vgl. dazu Hatt 1980, 60. - Binding 1993, 122.

110 Frey 2007b, 205-207.

111 FREY 2007b, 207.

112 So schon Jacobsthal 1944, 23. - In diesem Sinn auch Frey 1993, 155. coupées (Abb. 9/7). ${ }^{113}$ Das Tragen derartiger Schmuckstücke hätte im zweiten Fall wie der Besitz echter Trophäen die Kräfte der getöteten Feinde auf den Träger der Trophäe bzw. des Schmuckstückes übertragen. Ein allerdings erst spätlatènezeitlicher Anhänger mit dem Kopf eines Kelten aus Pocking unweit von Passau in Niederbayern (Abb. 13/2) $)^{114}$ - mit einem vergleichbaren Köpfchen von der Riegersburg in der Oststeiermark (Abb. 13/1), wenn auch ohne Öse ${ }^{115}$ - ist jedenfalls nur im Rahmen des Kopfkultes der Kelten zu erklären.
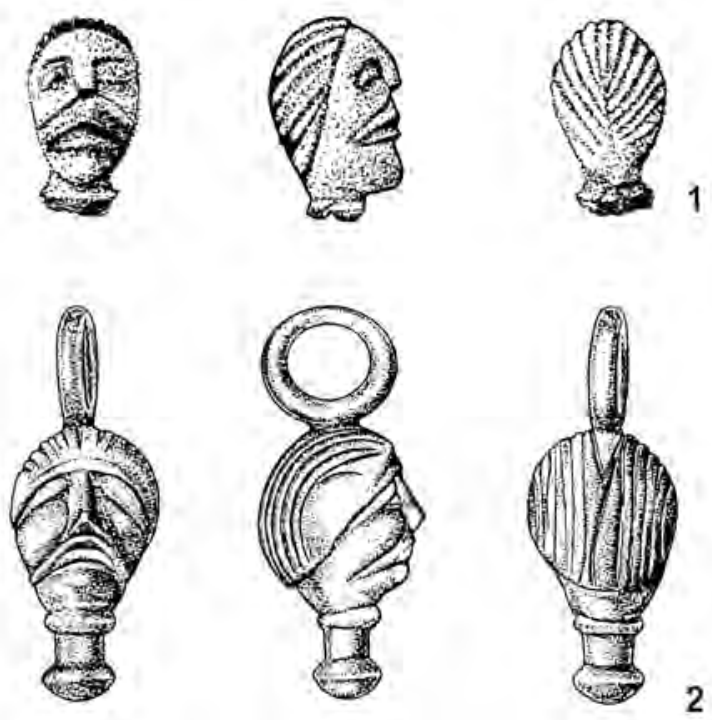

Abb. 13. Spätkeltische Anhänger in Form von têtes coupées. Bronze, Maßstab 1:1. - 1. Riegersburg (nach Kramer 1994, 333 und Abb. 2/1). - 2. Pocking (nach Kramer 1994, 333 und Abb. 2/2).

Marina Sarah Hess vermutet hinter den wiederkehrenden Motiven der frühkeltischen Kunst Chiffren, deren Botschaften sich uns entziehen. ${ }^{116}$ Menschliche Gesichter anstelle von vollständigen Körpern, die rar und ohne göttliche Attribute bleiben, hätten, sofern es sich um Elemente der Kleidung handelt, die am Körper getragen werden, wohl einen magischen oder apotropäischen Charakter gehabt. Anthropomorphe Gesichter, deren Vorbilder aus dem orientalischen oder etruskischen Raum stammen, stehen für eine Geschichte der schrittweisen Begegnung zwischen Mensch

113 LAmbrechts 1954, 22-25. - Binding 1993, 86, 95, 119-123. - So auch FREY 2002a, 178. - Zur archäologischen Überlieferung: MÜLLER 2002, 114, 129, 149-152.

114 Dannheimer, Gebhard 1993, 325/Nr. 411.

115 Kramer 1994.

116 Hess 2015b, bes. 331-336. 
und (mythologischem) Tier, einen Mythos, der dem latènezeitlichen Betrachter bekannt war, deren Botschaft sich der modernen Forschung aber nach wie vor entzieht.

Jean-Jacques Hatt hat in Bezug auf doppelköpfige Bilder in ersterem Sinn vorgeschlagen, diese als Darstellungen des Janus zu interpretieren und mit dem Gott Esus gleichzusetzen. ${ }^{117}$ Die bereits genannte janusartige, 2,30 $\mathrm{m}$ hohe frühlatènezeitliche Sandsteinstele aus Holzgerlingen in Baden-Württemberg ${ }^{118}$ dürfte jedenfalls einen heroisierten Ahnen mit einer Mistelblattkrone darstellen, der in „symmetrischer" Verdoppelung gleichsam in alle Richtungen schaut. Dem dürfte ein mittellatènezeitlicher zweigesichtiger Kalksteinkopf aus dem Heiligtum von Roquepertuse in Südfrankreich anzuschließen sein. ${ }^{119}$ Eine nicht näher bestimmbare frühlatènezeitliche Kleinbronze mit janusartigem Doppelgesicht und Mistelblattkrone stammt schließlich aus Lacoste bei Mouliets-et-Villemartin bei Bordeaux an der Atlantikküste. ${ }^{120}$

\section{Ergebnis}

Der bronzene Knotenarmreif mit zwei gegenständigen Kopfgruppen aus Möllbrücke datiert ins 5. Jh. v. Chr. Es handelt sich um einen südostalpinen Typus von Armreifen, dem die beiden gegenständigen, stark stilisierten Kopfgruppen eingefügt wurden. Der maskenhafte Gesichtsausdruck der Köpfe findet in der bronzenen „Maske“ aus dem Kröllkogel in Kleinklein eine frühe, etruskisch beeinflusste Parallele, noch aus der 1. Hälfte des 6. Jhs. v. Chr. Wie schon V. Megaw mit Blick auf vergleichbare frühlatènezeitliche Fingerringe aus Vače erwogen hat, könnte auch für das Aufkommen von Doppelgesichtern im Südostalpenraum im 5. Jh. v. Chr. ein direkter etruskischer Einfluss anzunehmen sein, auch wenn ein vergleichbarer Prozess der Darstellung von Köpfen bzw. Gesichtern („Masken“) auf zeitgleichem Ringschmuck der Kelten stattgefunden hat. Doch mag beides unabhängig voneinander auf etruskische Inspiration zurückzuführen sein, für den Südostalpenraum trotz nachgewiesener Kontakte zur Welt der Kelten nördlich der Alpen, zumal das Aufkommen von Appliken in Form von Köpfen bzw. Gesichtern („Masken“) im Südostalpenraum, verbunden mit dem Aufkommen der Situlenkunst und vielen anderen Neuerungen aus dem mediterranen Raum längst in massivem Umfang nachgewiesen ist. Inwieweit für die Deutung dieser Köpfe auf Ringschmuck apotropäische

117 Hatt 1980, 60. - Nicht ausgeschlossen bei Binding 1993, 122. 118 U. a. Frey 1993, 155 und Abb. 135. - Rieckhoff, Biel 2001, 191 mit Abb.

119 Dannheimer, Gebhard 1993, 344/Nr. 467c.

120 Sireix et al. 2002.
Vorstellungen zu vermuten sind oder an Darstellungen von Göttern und Heroen, die dann bei den Kelten jedenfalls Kronen aus Mistelblättern haben sollten, zu denken ist, oder auch an Dämonen, bleibt wohl eine kaum und nicht generell zu lösende Frage. Auch an eine Deutung als symbolische Darstellung von Köpfen von im Kampf getöteten Kriegern, têtes coupées, wurde gedacht. Den Köpfen (Gesichtern, „Masken“) fehlen jedenfalls jegliche nichtmenschliche Merkmale oder Attribute.

\section{Literatur}

APPLER 2006

H. APPLER, Ein frühlatènezeitliches Schmuckdepot vom Volderberg, Gem. Volders, Tirol, Tiroler Heimatblätter 81/1, 2006, 12-14.

Bagley 2013

J. M. BAgley, Potnia und Despotes Theron: transalpine Kontakte im Spiegel der Kunst der frühen Latènezeit, Archäologisches Korrespondenzblatt 43, 2013, 59-78.

BAgLeY 2014

J. M. Bagley, Zwischen Kommunikation und Distinktion: Ansätze zur Rekonstruktion frühlatènezeitlicher Bildpraxis. Vorgeschichtliche Forschungen 25, Rahden/Westf. 2014.

BIEL 1985

J. BieL, Der Keltenfürst von Hochdorf. Stuttgart 1985.

BINDING 1993

U. Binding, Studien zu den figürlichen Fibeln der Frühlatènezeit. Universitätsforschungen zur prähistorischen Archäologie 16, Bonn 1993.

BLÜMEL 2010

M. J. Blümel, Das Fürstengrab von Rodenbach. In: M. M. GreweNIG (Hrsg.), Die Kelten: Druiden. Fürsten. Krieger. Völklingen 2010, 152-153.

Božıč 1983

D. BožIČ (Hrsg.), KELTOI: Kelti in njihovi sodobniki na ozemlju Jugoslavije / Die Kelten und ihre Zeitgenossen auf dem Gebiet Jugoslawiens. Laibach 1983.

BožIČ 1999a

D. Božıč, Imenovali so se po Kolpi. In: Zakladi tisočletij. Laibach 1999, 174-176.

BožIČ $1999 b$

D. Božıč, Poljedelci in živinorejci v Posočju. In: Zakladi tisočletij. Laibach 1999, 177-180.

Božıč 2009

D. Božıč, A Hallstatt grave containing a cuirass, excavated near Stična by the Duchess of Mecklenburg in 1913: the reliability of grave groups from the Mecklenburg Collection, Arheološki vestnik 60, 2009, 63-95.

BožIČ 2010

D. Božıč, Zum Panzergrab von Stična und der Verlässlichkeit der Grabzusammenhänge in der Sammlung Mecklenburg, Acta Praehistorica et Archaeologica 42, 2010, 155-172.

Calzavara 1984

L. Calzavara, La zona pedemontana tra Brenta e Piave e il Cadore. In: A. Aspes (Hrsg.), Il Veneto nell'Antichità. Verona 1984, $847-866$.

Chieco Bianchi, Calzavara Capuis 1985

A. M. Chieco Bianchi, L. Calzavara Capuis, Este I: Le Necropoli Casa di Ricovero, Casa Muletti Prosdocimi e Casa Alfonsi. Monumenti Antichi 51, Rom 1985. 


\section{Corazza, Vitri 2001}

S. Corazza, S. Vitri, La Necropoli di Misincinis dopo lo Scavo: Primi Risultati delle Indagini 1995-1997. Udine 2001.

Cordie-Hackenberg 1992a

R. Cordie-Hackenberg, Tracht und Schmuck. In: Hundert Meisterwerke keltischer Kunst: Schmuck und Kunsthandwerk zwischen Rhein und Mosel. Schriftenreihe des Rheinischen Landesmuseums Trier 7, Trier 1992, 145-150.

Cordie-Hackenberg 1992b

R. Cordie-Hackenberg, Halsringe. In: Hundert Meisterwerke keltischer Kunst: Schmuck und Kunsthandwerk zwischen Rhein und Mosel. Schriftenreihe des Rheinischen Landesmuseums Trier 7, Trier 1992, 171-175.

Counts, Arnold 2010

D. B. Counts, B. Arnold (Hrsg.), The Master of Animals in Old World Iconography. Archaeolingua 24, Budapest 2010.

Crummy 2001

N. CRummy, Nail-cleaners from Britannia, Instrumentum 14, 2001 , 16.

DAL Ri 2012

L. DAL RI, La fibula semilunata con volto umano da Fiè/Völs, presso Bolzano, Archeologia Veneta 35, 2012, 148-161.

Dannheimer, GebHard 1993

H. Dannheimer, R. Gebhard (Hrsg.), Das keltische Jahrtausend. Mainz 1993.

Dular 1980

A. Dular, Die Bronzefigürchen aus Šmarjeta. In: Zbornik posvečen Stanetu Grabrovcu ob šestdestletnici. Situla 20/21, Laibach 1980, 263-266.

Dular 1999

J. DuLAR, Vače - središče na meji halštatskih skupnosti. In: Zakladi tisočletij. Laibach 1999, 116-119.

Dular 2003

J. Dular, Die hallstattzeitlichen Nekropolen in Dolenjsko. Opera Instituti Archaeologicae Sloveniae 6, Laibach 2003.

Еснт 1999

R. Еснт, Das Fürstinnengrab von Reinheim: Studien zur Kulturgeschichte der Früh-La Tène-Zeit. Saarbrücker Beiträge zur Altertumskunde 69, Bonn 1999.

EGG 1986a

M. EGG, Die „Herrin der Pferde“ im Alpengebiet, Archäologisches Korrespondenzblatt 16, 1986, 69-78.

EGG 1986b

M. EGG, Italische Helme. Monographien des Römisch-Germanischen Zentralmuseums 11, Mainz 1986.

EGG 1996

M. EGG, Das hallstattzeitliche Fürstengrab von Strettweg bei Judenburg in der Obersteiermark. Monographien des Römisch-Germanischen Zentralmuseums 37, Mainz 1996.

EGG 2013

M. EGG, Zur figuralen Kunst von Kleinklein. In: M. EGG, D. KRAMER (Hrsg.), Die hallstattzeitlichen Fürstengräber von Kleinklein in der Steiermark: der Kröllkogel. Monographien des RömischGermanischen Zentralmuseums 110, Mainz 2013, 447-472.

EGG, Munir 2013

M. EgG, J. Munir, Metallfunde. In: M. EgG, D. Kramer (Hrsg.), Die hallstattzeitlichen Fürstengräber von Kleinklein in der Steiermark: der Kröllkogel. Monographien des Römisch-Germanischen Zentralmuseums 110, Mainz 2013, 75-279.
EgG, Zeller 2005

M. EGG, K. W. ZeLLER, Zwei hallstattzeitlicher Grabkammern vom Dürrnberg bei Hallein - Befunde und Funde, Archäologisches Korrespondenzblatt 35, 2005, 345-360.

EGRI 2014

M. EGRI, Heads, masks and shifting identities: a note about some Danubian kantharoi with anthropomorphic decoration. In: C. Gosden, S. Crawford, K. Ulmschneider (Hrsg.), Celtic Art in Europe: Making Connections. Essays in Honour of Vincent Megaw on his $80^{\text {th }}$ Birthday. Oxford 2014, 73-85.

ENGELS 1972

H.-J. ENGELs, Der Fürstengrabhügel von Rodenbach. In: E. K. MoSER (Hrsg.), Festschrift für Otto Kleemann zum 60. Geburtstag. Bonner Hefte zur Vorgeschichte 3, Bonn 1972, 25-52.

FREY 1993

O.-H. Frey, Die Bilderwelt der Kelten. In: H. Dannheimer, R. Gebhard (Hrsg.), Das keltische Jahrtausend. Mainz 1993, 153-168.

FREY 1996

O.-H. Frey, Bemerkungen zu einigen Fundstücken der Frühlatènezeit aus Niederösterreich. In: E. Jerem, A. KrenN-LeEb, J.-W. Neugebauer, O.-H. Urban (Hrsg.), Die Kelten in den Alpen und an der Donau. Archaeolingua 1, Budapest - Wien 1996, 193-215.

FREY 2002a

O.-H. Frey, Die Fürstengräber vom Glauberg: Jenseitsvorstellungen und Bestattungsbrauchtum. In: H. Baitinger, B. Pinsker (Hrsg.), Das Rätsel der Kelten vom Glauberg: Glaube - Mythos - Wirklichkeit. Stuttgart 2002, 172-185.

FREY 2002b

O.-H. FreY, Frühe keltische Kunst - Dämonen und Götter. In: H. Baitinger, B. Pinsker (Hrsg.), Das Rätsel der Kelten vom Glauberg: Glaube - Mythos - Wirklichkeit. Stuttgart 2002, 186-205.

FREY 2007a

O.-H. FREY, Keltische Kunst in vorrömischer Zeit. Kleine Schriften aus dem Vorgeschichtlichen Seminar Marburg 57, Marburg/L. 2007.

FREY 2007b

O.-H. Frey, Die Erscheinungsform keltischer Götter in vorrömischer Zeit. In: H. Birkhan (Hrsg.), Kelten-Einfälle an der Donau. Denkschriften der Österreichischen Akademie der Wissenschaften, phil.-hist. Klasse 345, Wien 2007, 203-212.

Fürhacker, Pfeifer-Schäller, Wedenig 2004

R. FÜrhacker, I. Pfeifer-Schäller, R. Wedenig, Neue Erkenntnisse zu zwei Bronzeobjekten aus dem hallstattzeitlichen Gräberfeld Führholz bei Mittertrixen durch den Einsatz der industriellen 3D-Röntgen-Computertomografie, Rudolfinum 109, 2004, 109-120.

Gabrovec 1966

S. Gabrovec, Zur Mittellatènezeit in Slowenien, Arheološki vestnik $17,1966,33-106$.

Gabrovec 1987

S. Gabrovec, Jugoistočna regija sa zapadnom Panonijom. In: A. Benac (Hrsg.), Praistorija jugoslavenskih zemalja: 5. Željeznono doba. Sarajevo 1987, 25-181.

Gabrovec 1992

S. Gabrovec, Etruskischer Niederschlag in Slowenien. In: L. Aigner-Foresti (Hrsg.), Etrusker nördlich von Etrurien. 
Sitzungsberichte der Österreichischen Akademie der Wissenschaften, phil.-hist. Klasse 589, Wien 1992, 203-218.

GAMPER 2006

P. GAMPER, Die latènezeitliche Besiedlung am Ganglegg in Südtirol: Neue Forschungen zur Fritzens-Sanzeno-Kultur. Internationale Archäologie 91, Rahden/Westf. 2006.

Gangemi 2015

G. GANGemi, Considerazioni sulla necropoli di „Pian de la Gnela“. In: G. Gangemi, M. Bassetti, D. Voltolini (Hrsg.), Le Signore dell'Alpago: La Necropoli Preromana di “Pian de la Gnela” Pieve d'Alpago (Belluno). Treviso 2015, 175-178.

GAUER 1985

W. Gauer, Der Kessel von Hochdorf. In: Der Keltenfürst von Hochdorf. Methoden und Ergebnisse der Landesarchäologie. Stuttgart 1985, 124-129.

GLASER 1992

F. GLASER, Teurnia: Römerstadt und Bischofssitz. Klagenfurt 1992.

GLASER 2002

F. Glaser, Teurnia. In: M. ŠAšel Kos, P. Scherrer (Hrsg.), Die autonomen Städte in Noricum und Pannonien. Situla 40, Laibach 2002, 135-147.

GLeIRSCHER 1986

P. Gleirscher, Stilisierte Frauenfigürchen aus Südtirol und dem Trentino: „Reitia“, Göttin der „Räter“? Der Schlern 60, 1986, 178-190.

GLEIRSCHER 1993a

P. Gleirscher, Zum etruskischen Einfluss auf die Golaseccakultur und dessen Auswirkungen auf die Kulturverhältnisse im Alpenrheintal, Helvetia Archaeologica 24/1993-1994, 1993, 51-68.

GLEIRSCHER 1993b

P. Gleirscher, Zum etruskischen Fundgut zwischen Adda, Etsch und Inn, Helvetia Archaelogica 24/1993-1994, 1993, 69-105.

Gleirscher 2001

P. Gleirscher, Nuovi dati sulle fasi tardo hallstattiana e La Tène in Carinzia. In: S. Vitri, F. Oriolo (Hrsg.), I Celti in Carnia e nell'Arco Alpino Centro Orientale. Triest 2001, 211-226.

GLeirscher 2002a

P. Gleirscher, Die Kleinfunde vom Rungger Egg. In: P. GleirSCher, H. Nothdurfter, E. Schubert, Das Rungger Egg: Untersuchungen an einem eisenzeitlichen Brandopferplatz bei Seis am Schlern in Südtirol. Römisch-Germanische Forschungen 61, Mainz 2002, 36-172.

GLEIRSCHER 2002b

P. Gleirscher, Alpine Brandopferplätze. In: L. Zemmer-Plank, W. SöLder (Red.), Kult der Vorzeit in den Alpen: Opfergaben Opferplätze - Opferbrauchtum. Schriftenreihe der Arge Alp 10, Kommission I/Kultur und Gesellschaft, Bozen 2002, 591-634.

Gleirscher 2009

P. Gleirscher, Noreia - Atlantis der Berge: Neues zu Göttin, Stadt und Straßenstation. Klagenfurt - Laibach - Wien 2009.

Gleirscher 2011

P. Gleirscher, Die Hügelgräber von Frög: ein eisenzeitliches Herrschaftszentrum in Rosegg. Klagenfurt - Laibach - Wien 2011.

GLEIRSCHER 2018a

P. Gleirscher, Tagliaunghie dell'età del Ferro in area circumalpina. In: F. Nicolis, R. Oberosler (Hrsg.), Archeologia delle Alpi. Studi in onore di Gianni Ciurletti. Trient 2018, 59-68.

Gleirscher 2018b

P. Gleirscher, Karantanien: Slawisches Fürstentum und bairische Grafschaft. Klagenfurt 2018.
Guggisberg 2000

M. A. Guggisberg, Der Goldschatz von Erstfeld. Antiqua 32, Basel 2000.

Guggisberg 2010

M. A. Guggisberg, The mistress of animals, the master of animals: two complementary or oppositional religious concepts in early Celtic art? In: D. B. CounTs, B. Arnold (Hrsg.), The Master of Animals in Old World Iconography. Archaeolingua 24, Budapest 2010, 223-236.

Guggisberg, StÖllner 1996

M. A. Guggisberg, T. StöLlnER, Ein „Herr der Tiere“ im südlichen Ostalpenraum? Bemerkungen zur frühlatènezeitlichen Stellung einiger Neufunde aus dem Führholz bei Völkermarkt/Kärnten. In: T. STÖLLNER (Hrsg.), Europa celtica: Untersuchungen zur Hallstatt- und Latènekultur. Veröffentlichungen des Vorgeschichtlichen Seminars Marburg, Sonderband 10, Marburg 1996, 117-152.

GušTin 1980

M. GušTin, Ante Tubam / Der Hornbläser aus Idrija bei Bača. In: Zbornik posvečen Stanetu Grabrovcu ob šestdestletnici. Situla 20/21, Laibach 1980, 251-257.

HafFNER 1992

A. HAFFner, Zum Gürtel der Latènezeit. In: Hundert Meisterwerke keltischer Kunst: Schmuck und Kunsthandwerk zwischen Rhein und Mosel. Schriftenreihe des Rheinischen Landesmuseums Trier 7, Trier 1992, 151-158.

VON HASE 1973

F.-W. von HASE, Unbekannte frühetruskische Edelmetallfunde mit Maskenköpfen - Mögliche Vorbilder keltischer Maskendarstellungen. Hamburger Beiträge zur Archäologie 3, Hamburg 1973, $51-64$.

НАTт 1980

J.-J. НАтт, Die keltische Götterwelt und ihre bildliche Darstellung in vorrömischer Zeit. In: Die Kelten in Mitteleuropa (Ausstellungskatalog, Hallein 1980). Salzburg 1980, 52-67.

Hess 2015a

M. S. Hess, Bilder und Botschaften: Gesichtsdarstellungen der Frühlatènekunst. In: R. KARL, J. LesKovar (Hrsg.), Interpretierte Eisenzeiten: Fallstudien, Methoden, Theorie. Studien zur Kulturgeschichte Oberösterreichs 42, Linz 2015, 153-162.

Hess 2015b

M. S. Hess, Bilder und Botschaften: die Darstellung der Interaktion von Mensch und Tier als ein gemeinsames Moment der Frühlatènekunst, Praehistorische Zeitschrift 90, 2015, 318-338.

vON HOCHSTETTER 1883

F. von HochstetTer, Die neuesten Gräberfunde von Watsch und St. Margarethen in Krain und der Culturkreis der HallstätterPeriode. Denkschriften der Akademie der Wissenschaften, mathem.-naturwiss. Classe 47, Wien 1883, 161-210.

JABLONKA 2001

P. JablonKa, Die Gurina bei Dellach im Gailtal: Siedlung, Handelsplatz und Heiligtum. Aus Forschung und Kunst 33, Klagenfurt 2001.

Jacobsthal 1944

P. JaсовSthal, Early Celtic Art. Oxford 1944.

ЈоАснім 1992

H.-E. JoAснім, Goldringe. In: Hundert Meisterwerke keltischer Kunst: Schmuck und Kunsthandwerk zwischen Rhein und Mosel. Schriftenreihe des Rheinischen Landesmuseums Trier 7, Trier 1992, 179-183. 
Jung 2008

M. Jung, Palmettengesichter auf Attaschen: Etruskische Kannen als mögliche Vorbilder latènezeitlicher Gesichtsdarstellungen, Archäologisches Korrespondenzblatt 38, 2008, 211-222.

KNEZ 1986

T. Knez, Novo mesto I: Hallstattzeitliche Gräber. Carniola Archaelogica 1, Novo mesto 1986.

KoHLA 1973

F. X. KoHLA, Kärntens Burgen, Schlösser, Ansitze und wehrhafte Stätten. Aus Forschung und Kunst 17/1, Klagenfurt 1973.

Kossack 1993

G. Kossack, Hallstatt- und Latèneornament. In: H. Dannheimer, R. Gebhard (Hrsg.), Das keltische Jahrtausend. Mainz 1993, 138-168.

KOSSACK 2002

G. Kossack, Der zentrale Alpenraum während der Bronze- und vorrömischen Eisenzeit - Institutionen, Sachbesitz und religiöse Ausdrucksformen. In: L. Zemmer-Plank, W. Sölder (Red.), Kult der Vorzeit in den Alpen: Opfergaben - Opferplätze - Opferbrauchtum. Schriftenreihe der Arge Alp 10, Kommission I/ Kultur und Gesellschaft, Bozen 2002, 285-340.

KRAMER 1994

M. Kramer, Ein keltischer Kopf von der Riegersburg, BH Feldbach, Steiermark. In: C. Dовıат, H. W. Böнme (Hrsg.), Festschrift für Otto-Herman Frey zum 65. Geburtstag. Marburger Studien zur Vor- und Frühgeschichte 16, Marburg 1994, 331-338.

Krausse, Beilharz 2012

D. Krausse, D. Beilharz, Frühkeltische Machtzentren von Zentralfrankreich bis Böhmen. In: Die Welt der Kelten: Zentren der Macht - Kostbarkeiten der Kunst. Ostfildern 2012, 99-105.

Kromer 1974

K. Kromer, Ein Votivfigürchen aus Südtirol. Situla 14/15, Ljubljana 1974, 53-59.

LАмв 2019

A. W. LAмв, La Tène anthropoid art in Britain: changes in style and people, Archäologisches Korrespondenzblatt 49, 2019, 83-94.

LAMBRECHTS 1954

P. LAmbrechts, L'exaltation de la tête dans la pensée et dans l'art des Celtes. Brügge 1954.

LANG 2010

A. LANG, Reitia - Göttin der Räter. In: A. Schönweger (Hrsg.), Gott, weiblich. Bozen 2010, 17-26.

LOGAR 1980

N. Logar, Zwei vorgeschichtliche Gräber auf Ajdovski gradec bei Vranje. In: Zbornik posvečen Stanetu Grabrovcu ob šestdestletnici. Situla 20/21, Laibach 1980, 295-300.

Macellari 2003

R. Macellari, Il Sepolcreto Etrusco nel Terreno Arnoaldi di Bologna (550-350 a. C.). Bologna 2003.

Marzatico 2002

F. Marzatico, Die „Herrin der Pferde“. In: Archaeo Tirol, Kleine Schriften 4, Wattens 2002, 149-152.

Marzatico 2012

F. Marzatico, Testimonianze figurative nel bacino dell'Adige fra l'età del bronzo e l'età del Ferro, Preistoria Alpina 46, 2012, 309-332.

Megaw 1965-1966

J. V. S. Megaw, Two La Tène finger rings in the Victoria and Albert Museum, London: an essay on the human face und early Celtic art, Praehistorische Zeitschrift 43/44, 1965-1966, 96-166.
Megaw 1970

J. V.S. Megaw, Art of the European Iron Age: A Study of the Elusive Image. Bath 1970.

Megaw 2010

J.V.S. MEgAw, A world turned upside down: the bronze plaque from Stupava, okr. Malacky.In:J.ŠUtekovÁ,P. PAvúk, P. KALÁBKovÁ, B. KovÁr, (Hrsg.), PANTA RHEI: Studies on the Chronology and Cultural Development of South-Eastern and Central Europe in Earlier Prehistory. Presented to Juraj Pavúk on the Occasion of his $75^{\text {th }}$ Birthday. Studia Archaeologica et Mediaevalia 11, Bratislava 2010, 607-622.

Megaw, Megaw 1989

R. Megaw, J. V. S. Megaw, Celtic Art: From its Beginnings to the Book of Kells. London 1989.

Megaw, Megaw, Neugebauer 1989

J. V. S. Megaw, M. R. Megaw, J.-W. Neugebauer, Zeugnisse frühlatènezeitlichen Kunsthandwerks aus dem Raum Herzogenburg, Niederösterreich, Germania 67, 1989, 477-517.

VON MERHART 1932

G. von Merhart, Venetoillyrische Relieffigürchen aus Tirol, Mannus 24, 1932, 56-63.

MetZner-Nebelsick 2007

C. MetZner-Nebelsick, Pferdchenfibeln: Zur Deutung einer frauenspezifischen Schmuckform der Hallstatt und Frühlatènezeit. In: M. Blečić, M. Črešnar, B. Hänsel, A. Hellmuth, E. Kaiser, C. Metzner-Nebelsick (Hrsg.), Scripta praehistorica in honorem Biba Teržan. Situla 44, Laibach 2007, 707-735.

MetZner-Nebelsick, Nebelsick 1999

C. Metzner-Nebelsick, L. D. Nebelsick, Frau und Pferd - ein Topos am Übergang von der Bronze- zur Eisenzeit Europas, Mitteilungen der Anthropologischen Gesellschaft Wien 129, 1999, 69-106.

Moser 2010

S. Moser, Die Kelten am Dürrnberg: Eisenzeit am Nordrand der Alpen. Schriftenreihe des Keltenmuseums Hallein 1, Hallein 2010.

MÜLler 2002

F. MÜLLER, Götter - Gaben - Rituale: Religion der Frühgeschichte Europas. Mainz 2002.

Nascimbene 2015

A. Nascimbene, "Pian de la Gnela" nel contesto plavense e alpino. In: G. Gangemi, M. Bassetti, D. Voltolini (Hrsg.), Le Signore dell'Alpago: La Necropoli Preromana di “Pian de la Gnela” Pieve d'Alpago (Belluno). Treviso 2015, 167-170.

NoRTMANn 2016

H. Nortmann, Frühlatènekunst in der Mittelrheinzone: Charakter, Umfeld, Entstehung, Archäologisches Korrespondenzblatt 46, 2016, 213-231.

ORIOLO et al. 2015

F. Oriolo, G. Righi, A. Ruta Serafini, S. Vitri (Hrsg.), Celti sui Monti di Smeraldo. Triest 2015.

Pauli 1975

L. PAuli, Keltischer Volksglaube. Münchner Beiträge zur Vor- und Frühgeschichte 28, München 1975.

Pauli 1978

L. PAuli, Der Dürrnberg bei Hallein III. Münchner Beiträge zur Vor- und Frühgeschichte 18, München 1978.

Pavlovič 2014

D. Pavlovič, Drnovo. In: B. TeržAn, M. Črešnar (Hrsg.), Absolute Dating of the Bronze and Iron Ages in Slovenia. Catalogi et Monographiae 40, Laibach 2014, 491-504. 
Penninger 1972

E. Penninger, Der Dürrnberg bei Hallein I. Münchner Beiträge zur Vor- und Frühgeschichte 16, München 1972.

PetTarin 2006

S. Pettarin, Le necropoli di San Pietro al Natisone e Dernazzacco. Studi e ricerche di protostoria mediterranea 7, Rom 2006.

PRELOŽNiK 2007

A. Preložnik, Fibule Picene e Lucane nel Caput Adriae orientale. In: M. Guštin, P. Ettel, M. Buora (Hrsg.), Piceni ed Europa. Archeologia frontiera 6, Udine 2007, 123-134.

RiecKHOFF, BIEL 2001

S. Rieckhoff, J. Biel, Die Kelten in Deutschland. Stuttgart 2001.

Rossi 2005

F. Rossi, La Dea Sconosciuta e la Barca Solare. Mailand 2005.

Ruta Serafini 1998

M. A. Ruta Serafini, Situla in bronzo con appliques antropomorfe. In: U. Raffaelli (Hrsg.), Rame d'Arte dalla Preistoria al XX Secolo nelle Alpi Centro-Orientali. Trient 1998, 52-53.

SEIDL 2005

S. SEIDL, Una collezione di materiale piceno all'Università di Jena, Quaderni Friulani d'Archeologia 15, 2005, 57-67.

SIEGFRIED-WEIsS 1979

A. SiEgFried-Weiss, Der Ostalpenraum in der Hallstattzeit und seine Beziehungen zum Mittelmeergebiet. Hamburger Beiträge zur Archäologie 6, Hamburg 1979.

SiREIX et al. 2002

C. Sireix, M. Sireix, J. Gomez de Soto, M. Pernot, La tête janiforme à double feuille de Gui de La Tène ancienne de Lacoste à Mouliets-et-Villemartin (Gironde, France), Archäologisches Korrespondenzblatt 32, 2002, 81-89.

SORMAZ, STÖLlnER 2005

T. SORMAZ, T. STÖLLNER, Zwei hallstattzeitliche Grabkammern vom Dürrnberg bei Hallein - Neue dendrochronologische Ergebnisse zum Übergang von der Hallstattzeit zur Frühlatènezeit, Archäologisches Korrespondenzblatt 35, 2005, 361-376.

STARÈ 1953

F. STARÈ, Drei vorgeschichtliche Gräber aus dem Save-Anrain-gebiet, Arheološki vestnik 4, 1953, 264-281.

STÖLLNER 2002

T. STÖLLNER, Verloren, versteckt, geopfert? Einzeldeponate der Eisenzeit in alpinen Extremlagen und ihre bronzezeitlichen Wurzeln. In: L. Zemmer-Plank, W. Sölder (Red.), Kult der Vorzeit in den Alpen: Opfergaben - Opferplätze - Opferbrauchtum. Schriftenreihe der Arge Alp 10, Kommission I/Kultur und Gesellschaft, Bozen 2002, 567-589.

STÖLLNER 2010

T. STÖLLNER, Kontakt, Mobilität und Kulturwandel im Frühlatènekreis - das Beispiel Frühlatènegürtelhaken. In: E. JeREM, M. Schönfelder, G. Wieland (Hrsg.), Nord-Süd, Ost-West: Kontakte während der Eisenzeit in Europa. Budapest 2010, 277-319.

STROH 1935

A. SтRон, Frühlatènegrab von Schwieberdingen, OA, Ludwigsburg, Germania 19, 1935, 290-295.

SZILÁGYi 1992

J. G. SZilágyi, Transdanubien und Italien im 6.-5. Jh. In: L. AigneRForesti (Hrsg.), Etrusker nördlich von Etrurien. Sitzungsberichte der Österreichischen Akademie der Wissenschaften, phil.hist. Klasse 589, Wien 1992, 219-234.
TAPPERT 1994

C. TAPPERT, Frühlatènezeitliche Siedlungsfunde vom Aster Weg in Straubing, Jahresbericht des Historischen Vereins Straubing und Umgebung 96, 1994, 32-142.

Tecco Hvala 2012

S. Tecco Hvala, Magdalenska gora: Social structure und burial rites of the Iron Age community. Opera Instituti Archaeologici Sloveniae 26, Laibach 2012.

TERŽAN 1976

B. TeržAn, Die Certosafibel, Arheološki vestnik 27, 1976, 317-443. TERŽAN 1990

B. TeržAn, Halbmondfibeln, Arheološki vestnik 41, 1990, 49-88.

TERŽAN 1998

B. TERŽAN, Auswirkungen des skythisch geprägten Kulturkreises auf die hallstattzeitlichen Kulturgruppen Pannoniens und des Ostalpenraumes. In: B. Hänsel, J. Machnik (Hrsg.), Das Karpatenbecken und die osteuropäische Steppe. Prähistorische Archäologie in Südosteuropa 12, Rahden/Westf. 1998, 511-560.

TERŽAN 2009

B. TeržAn, Eine latèneartige Fremdform im hallstättischen Vače. In: G. Tiefengraber, B. Kavur, A. Gaspari (Hrsg.), Keltske študije II: Studies in Celtic Archaeology. Papers in honour of Mitja Guštin. Protohistoire Européenne 11, Montagnac 2009, 85-99.

TERŽAN 2011

B. TeržAn, Hallstatt Europe: some aspects of religion and social structure. In: G. R. Tsetskhladze (Hrsg.), The Black Sea, Greece, Anatolia and Europe in the First Millennium BC. Colloquiae Antiqua 1, Leuven - Paris - Walpole 2011, 233-264.

TeržAn im Druck

B. TERžAn, Early La Tène elements in the late south eastern Alpine Hallstatt culture - an outline. In: M. GUŠTin, W. DAvid (Hrsg.), Die Kelten im Südostalpenraum, im Druck.

TERŽAN, ČreŠnAR 2014

B. TeržAn, M. ČrešnAR, Attempt at an absolute dating of the Early Iron Age in Slovenia. In: B. TeRŽAN, M. ČrešNAR (Hrsg.), Absolute Dating of the Bronze and Iron Ages in Slovenia. Catalogi et Monographiae 40, Laibach 2014, 703-725.

TiefENGRABER 2015

G. Tiefengraber, Eisenzeit. In: B. Hebert (Hrsg.), Urgeschichte und Römerzeit in der Steiermark. Geschichte der Steiermark 1, Wien - Köln - Weimar 2015, 487-682.

Tomedi 2002

G. Tomedi, Zur Emblematik der späten Bronzezeit und der frühen Eisenzeit im alpinen Raum. In: L. Zemmer-PlanK, W. SÖLdeR (Red.), Kult der Vorzeit in den Alpen: Opfergaben - Opferplätze - Opferbrauchtum. Schriftenreihe der Arge Alp 10, Kommission I/Kultur und Gesellschaft, Bozen 2002, 1211-1235.

Turk et al. 2009

P. Turk, D. Božıč, J. Istenič, N. Osmuk, Ž. Šmit, New pre-Roman inscriptions from western Slovenia: the archaeological evidence. In: G. Tiefengraber, B. Kavur, A. Gaspari (Hrsg.), Keltske študije II: Studies in Celtic Archaeology. Papers in honour of Mitja Guštin. Protohistoire Européenne 11, Montagnac 2009, $47-64$.

VITRI 1997

S. VITRI, L'abitato di altura di Forgaria, Flagogna, loc. Castelvecchio (UD). In: L. Endrizzi, F. Marzatico (Hrsg.), Ori delle Alpi. Trient 1997, 580-583. 
VITRI 2001

S. VITRI, Lo stato della ricerca protostorica in Carnia. In: S. VitRI, F. Oriolo (Hrsg.), I Celti in Carnia e nell'Arco Alpino Centro Orientale. Triest 2001, 19-50.

VolToLini 2015

D. Voltolini, La voce dei centri planiziari protourbani. In: G. GANgemi, M. Bassetti, D. Voltolini (Hrsg.), Le Signore dell'Alpago: La Necropoli Preromana di “Pian de la Gnela” Pieve d'Alpago (Belluno). Treviso 2015, 171-174.

WeDENIG 1990

R. WeDENIG, Vorbericht über die Ausgrabung 1989 im hallstattzeitlichen Gräberfeld von Führholz in Unterkärnten, Carinthia I 180, 1990, 171-196.

WEDENIG 1994

R. WedEnig, Das hallstattzeitliche Gräberfeld von Führholz. Begleitheft zur Sonderausstellung in Völkermarkt, 1. Juni bis 18. Juni 1994. Völkermarkt 1994, 3-9.

WeDENIG 1997

R. WeDENIG, Vorbericht über die Ausgrabung 1996 im hallstattzeitlichen Gräberfeld von Führholz in Unterkärnten, Carinthia I 187, 1997, 65-93.

WEDENIG 1999

R. Wedenig, Das hallstattzeitliche Gräberfeld von Führholz in Unterkärnten, Archäologie Österreichs 10/2, 1999, 4-17.

WeDENIG 2005

R. WEDENIG, Zehn Jahre Gräberarchäologie in Führholz - eine kurze Übersicht. In: R. WeDENIG (Hrsg.), Hallstattkultur im Trixnertal (Begleitheft zur Ausstellung, Völkermarkt, Klagenfurt 2005). Völkermarkt 2005, 19-33.

WEISS 1993

R. M. WeIss, Der Brustpanzer von Stična-St. Veit, Acta Praehistorica et Archaeologica 25, 1993, 168-185.

WeLLS 1978

P. S. Wells, A bronze figurine from Stična in Slovenia, Archaeological News 7/4, 1978, 73-82.

WeLLS 1981

P. S. WeLLs, The Emergency of an Iron Age Economy: The Mecklenburg Grave Groups from Hallstatt and Stična. American School of Prehistoric Research Bulletin 33, Cambridge/Mass. 1981.

WeLLS 2012

P. S. Wells, How Ancient Europeans Saw the World. Princeton 2012.

\author{
Paul Gleirscher \\ Abteilung für Ur-und Frühgeschichte \\ Landesmuseum für Kärnten \\ Sammlungs- und Wissenschaftszentrum \\ Liberogasse 8 \\ 9020 Klagenfurt am Wörthersee \\ Österreich \\ paul.gleirscher@landesmuseum.ktn.gv.at \\ (D) orcid.org/0000-0002-7003-7963
}

